\title{
Bifurcation of magnetorheological film-substrate elastomers subjected to biaxial pre-compression and transverse magnetic fields
}

\author{
M. Rambausek, K. Danas* \\ LMS, C.N.R.S, École Polytechnique, Institut Polytechnique de Paris, Palaiseau, 91128, France
}

\begin{abstract}
This work investigates the primary sinusoidal bifurcation wrinkling response of single- and multi-layered magnetorheological elastomer (MRE) film-substrate systems subjected to combined transverse applied magnetic fields and in-plane biaxial pre-compression. A recently proposed continuum model that includes the volume fraction of softmagnetic particles is employed to analyze the effect of the magnetic properties upon the bifurcation response of the system. The analysis is built in a highly versatile manner using a finite-element discretization approach along the direction of the applied magnetic field and Fourier expansions along the infinite in-plane layer directions. This allows for a seamless investigation of various multi-layered structures. First, we analyze the effect of biaxial pre-compression upon the critical magnetic field for a film-substrate system and for various mechanical stiffness ratios. We observe a kink in the critical magnetic curves and a reflection in the corresponding wave numbers as they cross the equi-biaxial pre-compression regime. Subsequently, we consider a MRE film bonded to a MRE substrate and study the effect of the particle volume fraction ratios in those two parts. As a result, we obtain sharp pattern transitions, i.e., long to short wavelengths changes with only minor perturbations of the applied pre-compression. The presence of a magnetic substrate changes qualitatively and quantitatively the bifurcation response of the film/substrate system. Finally, we carry out a data-mining exercise to minimize the critical magnetic field at bifurcation by using three different topologies, i.e., a monolayer, a bilayer and a sandwich film. We find that the topologies resembling closely the monolayer one lead to the lowest critical magnetic fields for a given biaxial pre-compression.
\end{abstract}

Keywords: magneto-elasticity, magnetorheological elastomers, stability, finite elements, Fourier

\section{Introduction}

Recently, Psarra et al. (2019) observed experimentally and numerically that a thin magneto-elastic film bonded on a soft non-magnetic substrate exhibits wrinkling and crinkling instabilities under a combined magnetic loading and uniaxial pre-compression. In particular, the film was made from a magnetorheological elastomer (MRE) which consisted of a soft silicone (shear modulus in the order of a few $\mathrm{kPa}$ ) comprising iron particles at a volume fraction of $c^{f}=0.2$ (i.e. $20 \mathrm{vol} \%$ ). The non-trivial coupling between the magnetic and mechanical response led to interesting critical field modulation, which is otherwise impossible with purely mechanical loads alone.

That work was inspired by earlier investigations of magneto-mechanical instabilities on thin plates (Moon and Pao, 1969), rectangular beams (Kankanala, 2007) and surfaces (Otténio et al., 2008) as well as by more recent theoretical (Danas and Triantafyllidis, 2014) and experimental (Psarra et al., 2017, 2019) studies. In these works, the authors used either purely phenomenological functions for MREs with particle-chain microstructures or simple energy functions that had no particle-particle interactions included, therefore making very difficult the possibility to explore the effects

\footnotetext{
Cite as: Int. J. Non-Linear Mech. 128 (2021) 103608, https : //doi.org/10.1016/j .i jnonlinmec. 2020.103608

* Corresponding author Danas)

Email addresses: matthias.rambausek@polytechnique.edu (M. Rambausek), konstantinos.danas@polytechnique.edu (K.
} 
of the properties of the constituent phases (such as particle volume fraction, matrix stiffness, coupling effects etc). Both of these studies were also carried out under uniaxial plane-strain pre-compression loads.

In turn, by replacing iron with permanently magnetizable hard-magnetic particles (e.g. NdFeB) (Kalina et al., 2017; Keip and Sridhar, 2019), novel hard-magnetic magnetorheological elastomers (hMREs) have been employed for controlling the deformation of films (Kim et al., 2018, 2019; Zhao et al., 2019a). In the larger context of coupled surface pattern control, one may refer to Su et al. (2019), Liu et al. (2019), Zhao et al. (2019b) and Su et al. (2020).

In the context of pure mechanical loads, the classical problem of a thin film bonded to a soft substrate in mechanics (Biot, 1963; Allen, 1969) has shown a surprising richness in (secondary) instability-phenomena going beyond simple wrinkling such as complex surface-patterns (Chen and Hutchinson, 2004; Huang et al., 2005; Audoly and Boudaoud, 2008; Cai et al., 2011; Xu et al., 2014; Chakrabarti et al., 2018; Xu et al., 2020), creases (Huang et al., 2005; Cao and Hutchinson, 2012a; Hutchinson, 2013; Wang and Zhao, 2014; Fu and Ciarletta, 2015), ridges (Wang and Zhao, 2014) and folds (Pocivavsek et al., 2008; Sun et al., 2012; Cao and Hutchinson, 2012b). Similar phenomena occur in bending problems, where the non-uniformity of the deformation is the main ingredient (Destrade et al., 2009; Sigaeva et al., 2018) leading to the observed instabilities.

It is precisely this complexity in the mechanical setting as well as the availability of more advanced constitutive magneto-mechanical models (such as the ones proposed in Mukherjee et al. (2020)) that motivates us to go beyond the analysis of Danas and Triantafyllidis (2014) and Psarra et al. (2019). Specifically, we investigate the primary bifurcation problem of one or more magnetorheological elastomer layers bonded on passive or magnetorheological substrates using the coupled magneto-mechanical energy of Mukherjee et al. (2020) allowing to vary independently the constitutive properties of the matrix and the particles in the MRE materials. In this regard, we address in the present study the following aspects:

- general in-plane mechanical loading such as biaxial and equi-biaxial pre-compression,

- the complex magneto-mechanical interplay of material properties such as mechanical stiffness of the polymer phase and iron-particle volume fraction both in the film and the substrate,

- topology of the layers including multi-layered structures.

Specifically, we find interesting features resulting from the non-trivial magneto-mechanical coupling, such as sharp pattern transitions leading to significant wavelength amplitude increase with only minor changes of the applied precompression, as well as bi-modal regimes in the bifurcation diagrams for a MRE film on a MRE substrate. An additional important outcome of the study lies in the characterization of critical bifurcation states for biaxial pre-compressions and the transitions associated to the equi-biaxial states. While these key findings do not necessarily imply that the same exact patterns persist in the post-bifurcation regime, they, nevertheless, open up interesting perspectives regarding dynamic magneto-mechanical surface pattern switching and control. Furthermore, given the vast range of possibilities resulting from the numerous parameters and geometries that can be achieved, a detailed knowledge of such primary bifurcation points allows to systematically explore the post-bifurcation response numerically and design experimental studies similar to those carried out by Psarra et al. (2017). In order to limit the possible material responses to a handful of parameters that are also realizable experimentally, we focus in the present study on isotropic MREs without magnetic hysteresis. For this, we employ the material model presented recently by Mukherjee et al. (2020) for isotropic MREs, which includes explicitly the effect of the particle volume fraction, the mechanical properties of the polymeric matrix phase and the magnetic properties of the iron-particle phase. This model is based on earlier computational and analytical homogenization estimates (Lopez-Pamies et al., 2013; Lefèvre et al., 2017, 2020) and has been extensively calibrated.

Following this introduction, we present the fundamental theory, the governing variational principle and the generic stability problem as well as the its discrete counterpart in Section 2 using a combination of finite elements and Fourier expansions. Subsequently, Section 3 introduces the employed homogenization-guided continuum model for the MREs at hand. This is followed by three results sections. The first one, Section 4, is devoted to a thorough investigation of the critical bifurcation states of a single MRE layer on a passive substrate subjected to a transverse magnetic field and biaxial pre-compression. Section 5 extends these results to magnetic substrates revealing additional interesting effects. Section 6 is concerned with multilayer MRE films and the important question of the minimization of the critical magnetic field over a wide range of parameters for single- and multilayer film topologies. Finally, the study is concluded with Section 7. 


\section{Field equations, potential energy and bifurcation analysis}

In this study we consider non-dissipative magneto-elasticity at finite strains (Brown, 1966; Dorfmann and Ogden, 2003; Kankanala and Triantafyllidis, 2004; Bustamante et al., 2008) in absence of body forces and free currents. In a Lagrangian setting (coordinates $\mathbf{X}$ ) the governing equations are given as

$$
\operatorname{Div} \mathbf{B}=0, \quad \operatorname{Curl} \mathbf{H}(\mathbf{F}, \mathbf{B})=\mathbf{0}
$$

and

$$
\operatorname{Div} \mathbf{S}(\mathbf{F}, \mathbf{B})=\mathbf{0}, \quad \operatorname{Curl} \mathbf{F}=\mathbf{0}
$$

where $\mathbf{B}$ and $\mathbf{H}$ denote the magnetic field and the magnetic $h$-field in the reference configuration, $\mathbf{S}$ denotes the total first Piola-Kirchhoff stress and $\mathbf{F}$ is the deformation gradient. The former three are related to their Eulerian (coordinates x) counterparts via

$$
\mathbf{B}=J \mathbf{F}^{-1} \cdot \mathbf{b}, \quad \mathbf{H}=\mathbf{F}^{\mathrm{T}} \cdot \mathbf{h}, \quad \mathbf{S}=J \boldsymbol{J} \cdot \mathbf{F}^{-\mathrm{T}},
$$

with $\boldsymbol{J}=\operatorname{det} \mathbf{F}$ and $\boldsymbol{\sigma}$ being the total Cauchy-type stress. Moreover, we may express $\mathbf{B}$ and $\mathbf{F}$ in terms of the Lagrangian vector potential $\mathbf{A}$ and the deformation map $\varphi: \mathbf{x}=\varphi(\mathbf{X})$ as

$$
\mathbf{B}=\operatorname{Curl} \mathbf{A}, \quad \mathbf{F}=\operatorname{Grad} \boldsymbol{\varphi}
$$

such that (1) $)_{1}$ and (2) $)_{2}$ are fulfilled identically.

Subsequently, we consider an energy-density $W(\mathbf{F}, \mathbf{B}) \equiv W(\operatorname{Grad} \boldsymbol{\varphi}, \operatorname{Curl} \mathbf{A})$ per unit reference volume $\mathcal{V}$ such that the potential energy becomes

$$
\mathcal{P}(\boldsymbol{\varphi}, \mathbf{A})=\int_{\mathcal{V}} W(\operatorname{Grad} \boldsymbol{\varphi}, \operatorname{Curl} \mathbf{A}) \mathrm{d} V
$$

This leads to the variational problem

with

$$
\left\{\boldsymbol{\varphi}^{*}, \mathbf{A}^{*}\right\}=\arg \left\{\inf _{\boldsymbol{\varphi} \in \mathcal{K}_{\varphi}} \inf _{\mathbf{A} \in \mathcal{K}_{\mathbf{A}}} \mathcal{P}(\boldsymbol{\varphi}, \mathbf{A})\right\}
$$

$$
\begin{aligned}
& \mathcal{K}_{\varphi}=\left\{\varphi \mid \varphi_{i} \in \mathcal{H}^{1}, \varphi_{i}=\bar{\varphi}_{i} \text { on } \partial \mathcal{V}_{\varphi_{i}} \cup \mathcal{B}^{\varphi_{i}}\right\} \\
& \mathcal{K}_{\mathbf{A}}=\left\{\mathbf{A} \mid A_{i} \in \mathcal{H}^{1}, A_{i}=\bar{A}_{i} \text { on } \partial \mathcal{V}_{A_{i}}, \operatorname{Div} \mathbf{A}=0 \text { in } \mathcal{V}\right\} .
\end{aligned}
$$

Here, $\mathcal{V}$ denotes the domain of the boundary value problem comprising one or more bodies $\mathcal{B}^{(i)}$, as well as the air $\mathcal{V}^{\prime}=\mathcal{V} \backslash \bigcup_{i} \mathcal{B}^{i}$, which constitutes a magnetic material with vanishing mechanical properties. Prescribed boundary values are indicated with an overline. In turn, the constraint on Div A in (6c) describes the Coulomb gauge condition, which ensures uniqueness of the solution for $\mathbf{A}$ without affecting the results for $\mathbf{B}$. The stationary conditions in (6a) yield $(1)_{2}$ and $(2)_{1}$ as well as the constitutive relations

$$
\mathbf{H}=\frac{\partial W(\mathbf{F}, \mathbf{B})}{\partial \mathbf{B}}, \quad \mathbf{S}=\frac{\partial W(\mathbf{F}, \mathbf{B})}{\partial \mathbf{F}} .
$$

\subsection{The boundary value problem and the form of the principal solution}

We discuss now the actual geometry under consideration, which, in the more general case here, consists of a multilayered structure $\mathcal{B}=\bigcup_{i} \mathcal{B}^{i}$. All layers, denoted by $\mathcal{B}^{i}$, have uniform material properties and are stacked along the $X_{3}$ direction, while being of infinite lateral extent $\left(X_{1}, X_{2} \in(-\infty, \infty)\right)$, as shown in Figure 1. In addition, above and below the structure lies free air space $\mathcal{V}^{\prime}$. The homogeneous external magnetic field $\mathbf{b}^{\infty}=\left(0,0, b^{\infty}\right)$ is applied parallel to the out-of-plane $\left(-\infty<X_{3}<\infty\right)$ direction. This corresponds to distant magnets perpendicular to the layers, again with infinite lateral extent. The structure is furthermore subjected to in-plane stretches along $X_{1}$ and $X_{2}$ and 

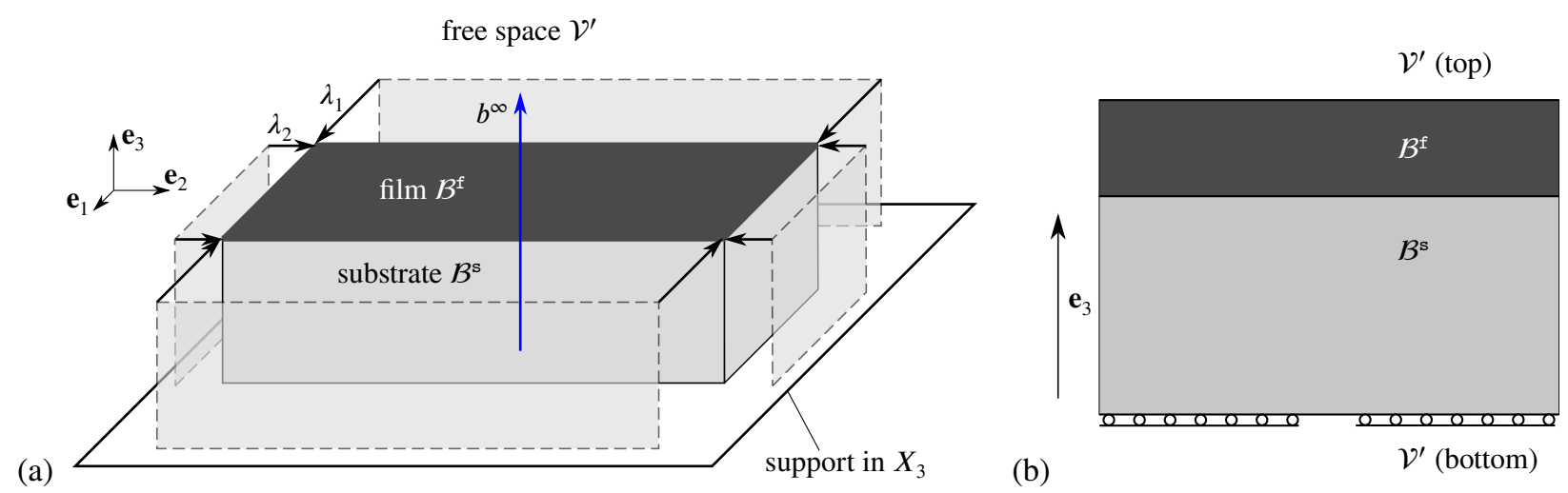

Figure 1: (a) The boundary value problem (BVP) consisting of a film bonded on a substrate. The film itself is also allowed to comprise more layers (see Fig. 2). The bottom part of the structure is fixed along the $X_{3}$ direction, while both the film and substrate extend to infinity in the lateral directions $X_{1}-X_{2}$ (not explicitly shown in the sketch). The magnetic field is applied along the direction $X_{3}$ and the surrounding air (not explicitly sketched) extends to $-\infty$ and $+\infty$. (b) Cross-section of the film-substrate-air system.

has an out-of-plane support at its bottom. At the interfaces between the structure and the free space (air) and between individual layers, we consider continuity of the displacement field and the traction vectors (Danas and Triantafyllidis, 2014). Note that we restrict the present study to isotropic materials but the proposed methodology is easily extended to anisotropic materials of any coupling, mechanical or not (see for example Danas et al. (2019)).

The form of the principal solution for the previously-described boundary value problem is domain-wise uniform ${ }^{1}$ and reads

$$
\begin{array}{llll}
\mathbf{F}=\lambda_{1} \mathbf{e}_{1} \otimes \mathbf{e}_{1}+\lambda_{2} \mathbf{e}_{2} \otimes \mathbf{e}_{2}+\lambda_{3} \mathbf{e}_{3} \otimes \mathbf{e}_{3} & \text { in } & \mathcal{B}^{i}, \\
\mathbf{B}=\lambda_{1} \lambda_{2} b^{\infty} \mathbf{e}_{3}, & \text { or } \quad \mathbf{b}=b^{\infty} \mathbf{e}_{3} & \text { in } & \mathcal{V},
\end{array}
$$

whereby $\lambda_{1}$ and $\lambda_{2}$ are constant throughout $\mathcal{B}$ because of the continuity of the displacement field. Since the air domain has vanishing mechanical properties, the actual value of $\mathbf{F}$ may take any form. A convenient choice that satisfies the continuity of displacements across the film/free-space interface is

$$
\mathbf{F}=\lambda_{1} \mathbf{e}_{1} \otimes \mathbf{e}_{1}+\lambda_{2} \mathbf{e}_{2} \otimes \mathbf{e}_{2}+1 \mathbf{e}_{3} \otimes \mathbf{e}_{3} \quad \text { in } \mathcal{V}^{\prime}
$$

This point is further detailed in Section 2.2.1.

The system of equations is closed by the previously-mentioned continuity of tractions across all interfaces. For the problem under consideration, there are no mechanical tractions along the direction $X_{3}$ throughout the entire structure. Thus, following, Kankanala and Triantafyllidis (2004) and Danas (2017), the vanishing Eulerian mechanical traction, $\mathbf{t}$, across an interface defined by a normal $\mathbf{n}$ separating two arbitrary materials (including air which is a magnetic material) is given by

$$
\mathbf{t}=\llbracket \boldsymbol{\sigma} \rrbracket \cdot \mathbf{n}=\mathbf{0} .
$$

Here, $\llbracket \boldsymbol{\sigma} \rrbracket$ is the jump of the total Cauchy stress along this interface. Using the continuity of the magnetic field $\mathbf{B}$ and the principal solution (8b), at the top and bottom free space $\mathcal{V}^{\prime}$ (air) in Fig. 1b, the total stress is simply equal to the Maxwell stress in air and thus reads

$$
\boldsymbol{\sigma}=\frac{1}{\mu_{0}} \mathbf{b} \otimes \mathbf{b}-\frac{|\mathbf{b}|^{2}}{2 \mu_{0}} \mathbf{1}=\frac{\left(b^{\infty}\right)^{2}}{\mu_{0}}\left(\mathbf{e}_{3} \otimes \mathbf{e}_{3}-\frac{1}{2} \mathbf{1}\right) \quad \text { in } \mathcal{V}^{\prime},
$$

with 1 denoting the second order identity tensor.

\footnotetext{
${ }^{1}$ This form of the principal solution is only valid if one assumes uniform material properties for each layer as well as material symmetries that maintain the corresponding geometric symmetries. That is the case in the present study where the materials used in each layer are isotropic.
} 
In turn, given the uniform principal solution for $\mathbf{F}$ in (8a), the total Cauchy stress in the film $\mathcal{B}^{\mathrm{f}}$ and the substrate $\mathcal{B}^{\text {s }}$ is also uniform and is given in terms of the energy densities $W(\mathbf{F}, \mathbf{B})$ by use of (3) and (7). Considering now the interface $\partial I^{\text {af }}=\mathcal{V}^{\prime} \cap \mathcal{B}^{f}$ with normal $\mathbf{n}=\mathbf{e}_{3}$ and using (10) leads to a single equation for $\lambda_{3}$ in the film, i.e.,

$$
\frac{\left(b^{\infty}\right)^{2}}{2 \mu_{0}}-\left.\frac{S_{33}\left(\lambda_{1}, \lambda_{2}, \lambda_{3}, b^{\infty}\right)}{\lambda_{1} \lambda_{2}}\right|_{\mathcal{B}^{f}}=0
$$

Again, $S_{33}$ is the component of the total first Piola-Kirchoff stress in the film as defined by (7). We recover a similar equation for $\lambda_{3}$ in the substrate $\mathcal{B}^{\mathrm{s}}$, by using the traction continuity either at the interface $\partial I^{\mathrm{fs}}=\mathcal{B}^{\mathrm{f}} \cap \mathcal{B}^{\mathrm{s}}$ or at $\partial I^{\mathrm{sa}}=\mathcal{B}^{\mathrm{s}} \cap \mathcal{V}^{\prime}$ with normal $\mathbf{n}=\mathbf{e}_{3}$, i.e., we get

$$
\frac{\left(b^{\infty}\right)^{2}}{2 \mu_{0}}-\left.\frac{S_{33}\left(\lambda_{1}, \lambda_{2}, \lambda_{3}, b^{\infty}\right)}{\lambda_{1} \lambda_{2}}\right|_{B^{s}}=0
$$

Remark 1. The above set of nonlinear algebraic equations may be solved analytically or numerically depending on the nonlinearity of the constitutive laws that are considered. In the present case, the solution for $\lambda_{3}$ is carried out numerically since no analytical solution is possible for the nonlinear magneto-mechanical constitutive laws considered in the following section. In addition, the same procedure can be readily expanded for any number of layers along the $X_{3}$ direction, as is the case in the present study (see Fig. 2).

\subsection{Bifurcation analysis}

In this section, we discuss first the bifurcation analysis for the given boundary value problem outlined previously and then present a novel numerical approach allowing to address the bifurcation problem using a combination of Fourier expansions and finite element discretization.

Of interest here is the stability of the principal solution $\mathbf{g}_{0}=\left\{\boldsymbol{\varphi}_{0}, \mathbf{A}_{0}\right\}=\mathbf{g}_{0}\left(b^{\infty}, \lambda_{1}, \lambda_{2}\right)^{2}$ presented in the previous section and more precisely the onset of first bifurcation. As explained earlier, the principal solution is obtained by minimizing the potential energy $\mathcal{P}$ in (5) with respect to the independent variables $\mathbf{g}$, i.e., by solving $\delta \mathcal{P} \equiv \mathcal{P}, \mathbf{g} \delta \mathbf{g}=0$. We also mention here that the applied loads involve the magnetic field as well as the two stretches independently, thus making the loading space three dimensional.

At relatively small values of the applied magnetic and mechanical loads, the principal solution $\mathbf{g}_{0}$ is stable, i.e. it is a local minimizer of the potential energy satisfying $\left(\mathcal{P}_{, \mathbf{g g}}\left(\mathbf{g}_{0}\right) \delta \mathbf{g}\right) \Delta \mathbf{g}>0$, for arbitrary perturbations $\Delta \mathbf{g} \neq \mathbf{0}$. As the applied loads increase, the film-substrate structure reaches a critical state, where the principal solution at hand $\mathbf{g}_{0}$ is no longer a local minimizer. Instead, non-uniform magnetic and mechanical fields may emerge in the layered structure. At that exact point, the second variation of the potential energy vanishes along a particular direction $\Delta \mathbf{g}$, which satisfies the condition:

$$
\Delta \delta \mathcal{P} \equiv\left(\mathcal{P}_{\mathbf{g g}}\left(\mathbf{g}_{0}\right) \delta \mathbf{g}\right) \Delta \mathbf{g}=\left.\int_{\mathcal{V}}\{\delta \boldsymbol{\varphi}, \delta \mathbf{A}\} \frac{\partial^{2} W(\operatorname{Grad} \boldsymbol{\varphi}, \operatorname{Curl} \mathbf{A})}{\partial\{\boldsymbol{\varphi}, \mathbf{A}\}^{2}}\right|_{\left\{\boldsymbol{\varphi}_{0}, \mathbf{A}_{0}\right\}}\{\Delta \boldsymbol{\varphi}, \Delta \mathbf{A}\} \mathrm{d} V=0 .
$$

Here, $\Delta \mathbf{g}$ are the bifurcation eigenmodes, and $\delta \mathbf{g}$ denote the arbitrary test functions corresponding to the independent variables of the problem, $\mathbf{g}$. Both $\Delta \mathbf{g}$ and $\delta \mathbf{g}$ are admissible and thus have to satisfy

$$
\begin{aligned}
\Delta \varphi(\mathbf{X}) & =\delta \varphi(\mathbf{X})=\mathbf{0} \quad \text { for } \quad \mathbf{X} \notin(\mathcal{B} \cup \partial \mathcal{B}), \\
\Delta \varphi_{3}(\mathbf{X}) & =\delta \varphi_{3}(\mathbf{X})=0 \quad \text { for } \quad X_{3}=0,
\end{aligned}
$$

and

$$
\begin{aligned}
\Delta \mathbf{A}(\mathbf{X}) & \rightarrow \mathbf{0}, \quad \delta \mathbf{A}(\mathbf{X}) \rightarrow \mathbf{0} \text { for } \quad X_{3} \rightarrow \pm \infty \\
\operatorname{Div} \Delta \mathbf{A} & =\operatorname{Div} \delta \mathbf{A}=0 \text { everywhere. }
\end{aligned}
$$

The first condition on $\Delta \varphi$ accounts for the fact that the energy in the free space is not affected by deformation such that $\Delta \varphi$ is basically arbitrary in that domain. By setting $\Delta \varphi$ (and also $\delta \varphi$ ) to zero in the free space we just remove spurious singular modes from the system. The second condition (15b) reflects the rigid vertical support (see Fig. 2) of the specimen.

\footnotetext{
${ }^{2}$ For simplicity in notation, we did not include the subscript 0 in the $\left\{b^{\infty}, \lambda_{1}, \lambda_{2}\right\}$ variables.
} 


\subsubsection{The discrete stability problem}

Due to the material homogeneity and infinite extent in the lateral directions $X_{1}-X_{2}$ of the layered structure in Fig. 1, we employ a Fourier expansion of the primary fields $\varphi$ and $\mathbf{A}$ in the $X_{1}-X_{2}$ plane. The Fourier expansion coefficients thus depend on $X_{3}$. In what follows, we denote the in-plane position vector as $\mathcal{X}=\left(X_{1}, X_{2}\right)$ and the wave vector as $\mathbf{\Omega}=\left(\Omega_{1}, \Omega_{2}\right)$.

For a continuous spectrum, the perturbations of the primary fields can be expressed ${ }^{3}$ as

$$
\begin{aligned}
& \Delta \boldsymbol{\varphi}(\mathbf{X})=\int_{\boldsymbol{\Omega}=\mathbf{0}}^{\infty} \Delta \boldsymbol{\varphi}^{\mathrm{C}}\left(\boldsymbol{\Omega}, X_{3}\right) \cos (\boldsymbol{\Omega} \cdot \mathcal{X})+\Delta \boldsymbol{\varphi}^{\mathrm{S}}\left(\boldsymbol{\Omega}, X_{3}\right) \sin (\boldsymbol{\Omega} \cdot \mathcal{X}) \mathrm{d} \boldsymbol{\Omega}, \\
& \Delta \mathbf{A}(\mathbf{X})=\int_{\boldsymbol{\Omega}=\mathbf{0}}^{\infty} \Delta \mathbf{A}^{\mathrm{C}}\left(\boldsymbol{\Omega}, X_{3}\right) \cos (\boldsymbol{\Omega} \cdot \mathcal{X})+\Delta \mathbf{A}^{\mathrm{S}}\left(\boldsymbol{\Omega}, X_{3}\right) \sin (\boldsymbol{\Omega} \cdot \mathcal{X}) \mathrm{d} \boldsymbol{\Omega} .
\end{aligned}
$$

Next, we discretize the above Fourier expansion coefficients in the $X_{3}$ direction by use of a finite element (FE) discretization. This FE discretization allows for the multiplicative decomposition of the Fourier coefficients into two factors, one depending on $X_{3}$ and the other on $\boldsymbol{\Omega}$.

Thus, let $\mathbf{N}^{i}\left(X_{3}\right)$ denote the individual finite element basis functions and $\left\{\Delta \hat{\boldsymbol{\varphi}}^{\mathrm{C}}(\mathbf{\Omega}), \Delta \hat{\boldsymbol{\varphi}}^{\mathrm{S}}(\mathbf{\Omega}), \Delta \widehat{\mathbf{A}}^{\mathrm{C}}(\mathbf{\Omega}), \Delta \widehat{\mathbf{A}}^{\mathrm{S}}(\mathbf{\Omega})\right\}$ the global finite element degrees of freedom per Fourier mode $\Omega$. Then, we have for $\Delta \varphi^{\mathrm{C}}, \Delta \varphi^{\mathrm{S}}, \Delta \mathrm{A}^{\mathrm{C}}$ and $\Delta \mathbf{A}^{\mathrm{S}}$ the discrete counterparts (indicated with a subscript $h$ )

$$
\begin{aligned}
& \Delta \boldsymbol{\varphi}_{h}^{\mathrm{C}}\left(\boldsymbol{\Omega}, X_{3}\right)=\sum_{i=1}^{N} \mathbf{N}^{i}\left(X_{3}\right) \cdot \Delta \widehat{\boldsymbol{\varphi}}^{\mathrm{C}}(\boldsymbol{\Omega})=\widehat{\mathbf{N}}\left(X_{3}\right) \cdot \Delta \widehat{\boldsymbol{\varphi}}^{\mathrm{C}}(\boldsymbol{\Omega}) \\
& \Delta \boldsymbol{\varphi}_{h}^{\mathrm{S}}\left(\boldsymbol{\Omega}, X_{3}\right)=\sum_{i=1}^{N} \mathbf{N}^{i}\left(X_{3}\right) \cdot \Delta \hat{\boldsymbol{\varphi}}^{\mathrm{S}}(\boldsymbol{\Omega})=\widehat{\mathbf{N}}\left(X_{3}\right) \cdot \Delta \hat{\boldsymbol{\varphi}}^{\mathrm{S}}(\boldsymbol{\Omega}) \\
& \Delta \mathbf{A}_{h}^{\mathrm{C}}\left(\boldsymbol{\Omega}, X_{3}\right)=\sum_{i=1}^{N} \mathbf{N}^{i}\left(X_{3}\right) \cdot \Delta \widehat{\mathbf{A}}^{\mathrm{C}}(\boldsymbol{\Omega})=\widehat{\mathbf{N}}\left(X_{3}\right) \cdot \Delta \widehat{\mathbf{A}}^{\mathrm{C}}(\boldsymbol{\Omega}) \\
& \Delta \mathbf{A}_{h}^{\mathrm{S}}\left(\boldsymbol{\Omega}, X_{3}\right)=\sum_{i=1}^{N} \mathbf{N}^{i}\left(X_{3}\right) \cdot \Delta \widehat{\mathbf{A}}^{\mathrm{S}}(\boldsymbol{\Omega})=\widehat{\mathbf{N}}\left(X_{3}\right) \cdot \Delta \widehat{\mathbf{A}}^{\mathrm{S}}(\boldsymbol{\Omega}) .
\end{aligned}
$$

Here, $\hat{\mathbf{N}}\left(X_{3}\right)$ collects all $\mathbf{N}^{i}\left(X_{3}\right)$ in an appropriate way. Next, we make the transition to a finite element notation for which we gather the finite element basis functions $\widehat{\mathbf{N}}\left(X_{3}\right)$ and the trigonometric terms "cos" and "sin" such that

$$
\begin{aligned}
& \Delta \boldsymbol{\varphi}_{h}(\mathbf{X})=\int_{\boldsymbol{\Omega}=\mathbf{0}}^{\infty} \underbrace{\left(\begin{array}{cc}
\hat{\mathbf{N}}\left(X_{3}\right) \cos (\boldsymbol{\Omega} \cdot \mathcal{X}) & \mathbf{0} \\
\mathbf{0} & \hat{\mathbf{N}}\left(X_{3}\right) \sin (\boldsymbol{\Omega} \cdot \mathcal{X})
\end{array}\right)}_{\check{\mathbf{N}}\left(\boldsymbol{\Omega}, \mathcal{X}, X_{3}\right)} \cdot \underbrace{\left(\begin{array}{c}
\Delta \hat{\boldsymbol{\varphi}}^{\mathrm{C}}(\boldsymbol{\Omega}) \\
\Delta \hat{\boldsymbol{\varphi}}^{\mathrm{S}}(\boldsymbol{\Omega})
\end{array}\right)}_{\Delta \check{\boldsymbol{\varphi}}(\boldsymbol{\Omega})} \mathrm{d} \boldsymbol{\Omega} \\
& =\int_{\boldsymbol{\Omega}=\mathbf{0}}^{\infty} \check{\mathbf{N}}\left(\boldsymbol{\Omega}, \mathcal{X}, X_{3}\right) \cdot \Delta \check{\boldsymbol{\varphi}}(\boldsymbol{\Omega}) \mathrm{d} \boldsymbol{\Omega} .
\end{aligned}
$$

Analogously, one has

$$
\Delta \mathbf{A}_{h}(\mathbf{X})=\int_{\boldsymbol{\Omega}=\mathbf{0}}^{\infty} \check{\mathbf{N}}\left(\boldsymbol{\Omega}, \mathcal{X}, X_{3}\right) \cdot \Delta \check{\mathbf{A}}(\mathbf{\Omega}) \mathrm{d} \boldsymbol{\Omega} .
$$

\footnotetext{
${ }^{3}$ The same expansions are applied to $\{\delta \boldsymbol{\varphi}, \delta \mathbf{A}\}$. Also note that for the purpose of the stability analysis it is quite common to employ the complexvalued version of the Fourier transform or expansion. However, we developed our code mainly based on the real-valued formulation as given above. This allowed us to verify our code with the method of manufactured solutions in a straight-forward way.
} 
Given the matrix $\check{\mathbf{N}}$, we introduce next the matrices $\check{\mathbf{G}}$, $\check{\mathbf{C}}$ and $\check{\mathbf{D}}$ such that

$$
\begin{aligned}
& \check{\mathbf{G}}\left(\boldsymbol{\Omega}, \mathcal{X}, X_{3}\right)=\operatorname{Grad} \check{\mathbf{N}}\left(\boldsymbol{\Omega}, \mathcal{X}, X_{3}\right) \\
& \check{\mathbf{C}}\left(\boldsymbol{\Omega}, \mathcal{X}, X_{3}\right)=\operatorname{Curl} \check{\mathbf{N}}\left(\boldsymbol{\Omega}, \mathcal{X}, X_{3}\right)
\end{aligned}
$$

and

$$
\check{\mathbf{D}}\left(\boldsymbol{\Omega}, \mathcal{X}, X_{3}\right)=\operatorname{Div} \check{\mathbf{N}}\left(\boldsymbol{\Omega}, \mathcal{X}, X_{3}\right) .
$$

Each of these matrices can be additively decomposed as

$$
\begin{aligned}
& \check{\mathbf{G}}=\check{\mathbf{G}}^{\mathrm{C}}\left(\boldsymbol{\Omega}, \mathcal{X}, X_{3}\right) \cos (\boldsymbol{\Omega} \cdot \mathcal{X})+\check{\mathbf{G}}^{\mathrm{S}}\left(\boldsymbol{\Omega}, \mathcal{X}, X_{3}\right) \sin (\boldsymbol{\Omega} \cdot \mathcal{X}) \\
& \check{\mathbf{C}}=\check{\mathbf{C}}^{\mathrm{C}}\left(\boldsymbol{\Omega}, \mathcal{X}, X_{3}\right) \cos (\boldsymbol{\Omega} \cdot \mathcal{X})+\check{\mathbf{C}}^{\mathrm{S}}\left(\boldsymbol{\Omega}, \mathcal{X}, X_{3}\right) \sin (\boldsymbol{\Omega} \cdot \mathcal{X}) \\
& \check{\mathbf{D}}=\check{\mathbf{D}}^{\mathrm{C}}\left(\boldsymbol{\Omega}, \mathcal{X}, X_{3}\right) \cos (\boldsymbol{\Omega} \cdot \mathcal{X})+\check{\mathbf{D}}^{\mathrm{S}}\left(\boldsymbol{\Omega}, \mathcal{X}, X_{3}\right) \sin (\boldsymbol{\Omega} \cdot \mathcal{X}) .
\end{aligned}
$$

Using the above definitions and by virtue of the $L_{2}$-orthogonality of trigonometric functions, we reformulate the bifurcation criterion (14) in terms of the discrete fields as

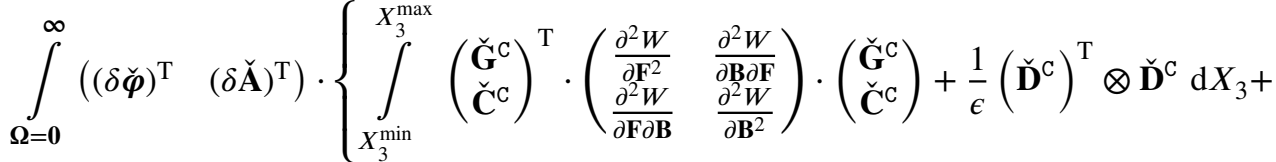

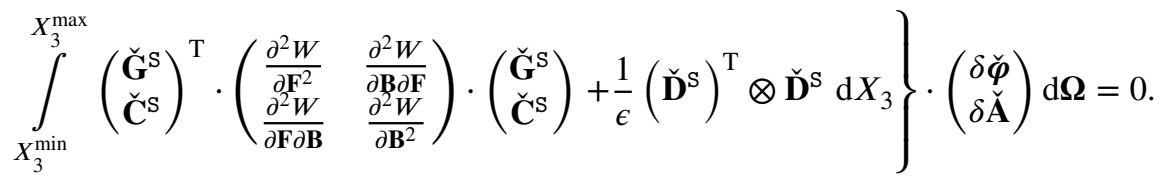

A few comments are in order for the transition from (14) to (29): First, we note that the constant resulting from the non-vanishing in-plane integration of the corresponding trigonometric functions has been normalized to one, which renders (29) an expression of density with respect to lateral area. This avoids the problem of an infinite energy ${ }^{4}$ that would result from (14) due to infinite lateral extents of the structure. Second, by contrast to the continuous problem, the domain of the integral in $X_{3}$ direction in (29) is not $\mathbb{R}$ but $\left(X_{3}^{\min }, X_{3}^{\max }\right)$. This "truncated" domain has to be large enough for a sufficient resolution of the magnetic field perturbations far from the specimen. Third, the fraction $1 / \epsilon$ is the "weight" of the penalty terms used to enforce $\operatorname{Div} \delta \mathbf{A}=0$. A value of $\epsilon=10^{-4}$ is found to be sufficient for the present purposes. Note further that contrary to the usual two- and three-dimensional finite element discretizations, these penalty terms do not need to be under-integrated in the present 1D FE problem. The same applies to the volumetric energy contributions in the context of quasi-incompressible materials, as will be used later. Throughout the present work we employ Lagrange-type finite elements of polynomial degree two.

In (29), the quantity inside the curly braces defines the finite element "stiffness" matrix of the system, denoted compactly by $\mathbf{K}\left(\boldsymbol{\Omega}, \mathbf{g}_{0}\right) \equiv \mathbf{K}\left(\boldsymbol{\Omega}, \boldsymbol{\varphi}_{0}, \mathbf{A}_{0}\right) \equiv \mathbf{K}\left(\boldsymbol{\Omega}, b^{\infty}, \lambda_{1}, \lambda_{2}\right)$. It is noted that the dependence of $\mathbf{K}$ on $\boldsymbol{\Omega}$ enters only via $\breve{\mathbf{G}}^{\mathrm{CIS}}, \breve{\mathbf{C}}^{\mathrm{CIS}}$ and $\check{\mathbf{D}}^{\mathrm{CIS}}$. By the short-hands $\delta \check{\mathbf{g}}(\boldsymbol{\Omega})=\{\delta \check{\boldsymbol{\varphi}}(\boldsymbol{\Omega}), \delta \check{\mathbf{A}}(\boldsymbol{\Omega})\}$ and $\Delta \check{\mathbf{g}}(\boldsymbol{\Omega})=\{\Delta \check{\boldsymbol{\varphi}}(\boldsymbol{\Omega}), \Delta \check{\mathbf{A}}(\boldsymbol{\Omega})\}$, we collect the degrees of freedom of the eigenmodes and test functions, respectively, such that (29) compactly reads

$$
\int_{\boldsymbol{\Omega}=\mathbf{0}}^{\infty}[\delta \check{\mathbf{g}}(\boldsymbol{\Omega})]^{\mathrm{T}} \cdot \mathbf{K}\left(\boldsymbol{\Omega}, \mathbf{g}_{0}\right) \cdot \Delta \check{\mathbf{g}}(\boldsymbol{\Omega}) \mathrm{d} \boldsymbol{\Omega}=0 .
$$

For a given principal solution $\mathbf{g}_{0}$ and depending on the properties of $\mathbf{K}$, equation (30) can be fulfilled under the following conditions ${ }^{5}$, of which the first is given by

$$
[\delta \check{\mathbf{g}}(\boldsymbol{\Omega})]^{\mathrm{T}} \cdot \mathbf{K}\left(\boldsymbol{\Omega}, \mathbf{g}_{0}\right) \cdot \Delta \check{\mathbf{g}}(\boldsymbol{\Omega})>0 \quad \forall \boldsymbol{\Omega} \in[\mathbf{0}, \boldsymbol{\infty}) \wedge \forall \Delta \check{\mathbf{g}}, \delta \check{\mathbf{g}} \neq \mathbf{0}
$$

\footnotetext{
${ }^{4}$ This is normally not an issue for bodies of finite extent for which the fields decrease with distance such that the overall energy remains finite. However, in the case of a periodic domain, which is infinite by construction, one has to provide an energy divided by some reference length, area or volume, respectively.

${ }^{5}$ This is a standard argument (see, e.g., Triantafyllidis and Needleman (1980)) and thus often omitted. We nevertheless present it for completeness.
} 
that is, $\mathbf{K}\left(\boldsymbol{\Omega}, \mathbf{g}_{0}\right)$ is positive definite. Then we must have $\Delta \check{\mathbf{g}}(\mathbf{\Omega})=\mathbf{0} \forall \boldsymbol{\Omega}$. The second case is characterized by

$$
[\delta \check{\mathbf{g}}(\mathbf{\Omega})]^{\mathrm{T}} \cdot \mathbf{K}\left(\boldsymbol{\Omega}, \mathbf{g}_{0}\right) \cdot \Delta \check{\mathbf{g}}(\boldsymbol{\Omega}) \geq 0 \quad \forall \boldsymbol{\Omega} \in[\mathbf{0}, \boldsymbol{\infty}) \wedge \forall \Delta \check{\mathbf{g}}, \delta \check{\mathbf{g}} \neq \mathbf{0}
$$

such that

$$
\exists \boldsymbol{\Omega}^{\mathrm{c}}: \min _{\Delta \check{\mathbf{g}}\left(\boldsymbol{\Omega}^{\mathrm{c}}\right) \neq \mathbf{0}}\left[\Delta \check{\mathbf{g}}\left(\boldsymbol{\Omega}^{\mathrm{c}}\right)\right]^{\mathrm{T}} \cdot \mathbf{K}\left(\boldsymbol{\Omega}^{\mathrm{c}}, \mathbf{g}_{0}\right) \cdot \Delta \check{\mathbf{g}}\left(\boldsymbol{\Omega}^{\mathrm{c}}\right)=0
$$

that is, $\mathbf{K}\left(\mathbf{\Omega}^{\mathrm{c}}, \mathbf{g}^{\mathrm{c}}\right) \equiv \mathbf{K}\left(\mathbf{\Omega}^{\mathrm{c}}, b^{\mathrm{c}}, \lambda_{1}^{\mathrm{c}}, \lambda_{2}^{\mathrm{c}}\right)$ is positive semi-definite. In that case the direction of $\Delta \check{\mathbf{g}}\left(\mathbf{\Omega}^{\mathrm{c}}\right)$ is determined, but not the magnitude. Thus, we have a nontrivial solution to (30). In the case that the integrand of (30) is less than zero for some $\boldsymbol{\Omega}$, the positive integrands can be compensated by the negative ones such that the integral still vanishes. This situation is already part of the post-bifurcation regime and thus beyond the scope of the present study.

In the present case, we minimize the smallest eigenvalue of $\mathbf{K}\left(\boldsymbol{\Omega}, \mathbf{g}_{0}\right)$ over $\boldsymbol{\Omega}$, which we denote with $\bar{\Lambda}^{\min }\left(b^{\mathrm{c}}, \lambda_{1}^{\mathrm{c}}, \lambda_{2}^{\mathrm{c}}\right)$ such that a critical state $\mathbf{g}^{\mathrm{c}} \equiv\left\{b^{\mathrm{c}}, \lambda_{1}^{\mathrm{c}}, \lambda_{2}^{\mathrm{c}}\right\}$ is defined by

$$
\bar{\Lambda}^{\min }\left(b^{\mathrm{c}}, \lambda_{1}^{\mathrm{c}}, \lambda_{2}^{\mathrm{c}}\right)=\min _{\boldsymbol{\Omega}}\left\{\min _{\Delta \check{\mathbf{g}}(\boldsymbol{\Omega}) \neq \mathbf{0}}[\Delta \check{\mathbf{g}}(\mathbf{\Omega})]^{\mathrm{T}} \cdot \frac{\mathbf{K}\left(\boldsymbol{\Omega}, b^{\mathrm{c}}, \lambda_{1}^{\mathrm{c}}, \lambda_{2}^{\mathrm{c}}\right)}{\|\Delta \check{\mathbf{g}}(\boldsymbol{\Omega})\|^{2}} \cdot \Delta \check{\mathbf{g}}(\boldsymbol{\Omega})\right\}=0,
$$

where the normalization by $\|\Delta \check{\mathbf{g}}\|^{2}$ is introduced to obtain a well defined eigenvalue problem with eigenvectors of unit magnitude. In a more descriptive manner, given a set of stretches $\lambda_{1}$ and $\lambda_{2}$, (34) can be understood as an equation for the critical field $b^{\mathrm{c}}\left(\lambda_{1}, \lambda_{2}\right)$. For this reason, in the following results sections, we use the superscript " ()$^{\mathrm{c}}$ " denoting "critical" only for $b^{c}$ but not to $\lambda_{1}$ and $\lambda_{2}$. Further details on the boundary conditions for the discrete problem, the underlying algorithm and the procedure for the identification of critical states are provided in Appendix B. The resulting implementation has undergone rigorous comparison with Hutchinson (2013) and Audoly and Boudaoud (2008) in the purely mechanical setting. Moreover, for the case of isotropic monolayer structures, our code has been benchmarked against the one underlying the work of Danas and Triantafyllidis (2014).

\section{Constitutive models and geometric parameters}

In this section, we specify the constitutive magneto-mechanical models that we will use to resolve the previously discussed bifurcation problem. The magnetorheological elastomers (MREs) considered in the present study consist of randomly distributed magnetic (e.g. iron) particles (denoted as phase $\mathrm{p}$ ) in an elastomeric matrix (denoted as phase $\mathrm{m}$ ). The distribution of the particles is assumed to be uniform and isotropic such that the overall response of the composite material is isotropic. The magnetic response of the particles is idealized to be free of hysteresis, which is a perfectly acceptable assumption for iron particles (Danas et al., 2012). The formulation of an appropriate macroscopic material model for MREs is a difficult problem (Javili et al., 2013; Keip and Rambausek, 2017) and still under active research. In the present study, we employ a continuum model (Mukherjee et al., 2020) that is guided by numerical (Danas, 2017) and analytical homogenization (Lopez-Pamies et al., 2013; Lefèvre et al., 2017). Due to its nature, the model features the particle volume fraction $c$ as a direct macroscopic material parameter. For completeness, we summarize the employed model in the context of quasi-incompressible media.

\subsection{An analytical continuum model for isotropic MREs}

Following Mukherjee et al. (2020), the energy density per unit referential volume of the MRE has four contributions

$$
W(\mathbf{F}, \mathbf{B})=\Psi^{\text {mech }}\left(I_{1}, J\right)+\Psi^{\operatorname{mag}}\left(J, I_{5}\right)+\Psi^{\text {couple }}\left(J, I_{5}, I_{6}\right)+\Psi^{\mathrm{vac}}\left(J, I_{5}\right)
$$

where the magneto-mechanical invariants are defined as

$$
\begin{aligned}
I_{1} & =\mathbf{F}: \mathbf{F}=\operatorname{tr} \mathbf{C}, \\
J & =\operatorname{det} \mathbf{F}=\sqrt{I_{3}}=\sqrt{\operatorname{det} \mathbf{C}}, \\
I_{5} & =\mathbf{C}:(\mathbf{B} \otimes \mathbf{B}), \\
I_{6} & =(\mathbf{C} \cdot \mathbf{B}) \cdot(\mathbf{C} \cdot \mathbf{B}) .
\end{aligned}
$$


Here, $\mathbf{C}=\mathbf{F}^{\mathrm{T}} \cdot \mathbf{F}$ is the right Cauchy-Green tensor. For the purely mechanical contribution $\Psi^{\text {mech }}\left(I_{1}, J\right)$, we use a quasi-incompressible variant of the family of models proposed by Lopez-Pamies et al. (2013), which are valid and explicit for any incompressible matrix phase that can be described by an energy density depending on $I_{1}$ (and $J$ in the quasi-incompressible approximation). In the present study, for simplicity, we use a Neo-Hookean description for the polymeric matrix phase, which by addition of iron particles (assumed as mechanically rigid) results in a continuum description of the energy density of the MRE that reads

$$
\Psi^{\text {mech }}\left(I_{1}, J\right)=\frac{G_{\mathrm{m}}}{2(1-c)^{5 / 2}}\left[I_{1}-3-2 \ln J\right]+\frac{G_{\mathrm{m}}^{\prime}}{2(1-c)^{6}}(J-1)^{2} .
$$

In this expression, $G_{\mathrm{m}}$ and $G_{\mathrm{m}}^{\prime}$ denote the shear and bulk moduli of the underlying matrix phase of the MRE. It is noted that the proposed compressible part of the model is sufficiently robust for the quasi-incompressible responses considered here. Specifically, we will set henceforth $G_{\mathrm{m}}^{\prime}=10^{5} G_{\mathrm{m}}$ for all materials considered in the present study which corresponds to a quasi-incompressible response.

In turn, the purely magnetic contribution to the MRE response reads as (Mukherjee et al., 2020)

$$
\Psi^{\operatorname{mag}}\left(J, I_{5}\right)=-\frac{\chi}{2 \mu_{0}(1+\chi)} \frac{I_{5}}{J^{2}}{ }_{2} \mathcal{F}_{1}\left[k, \frac{2}{k}, 1+\frac{2}{k},-\left(\frac{\chi}{\mu_{0}(1+\chi) m^{\mathrm{s}}} \frac{\sqrt{I_{5}}}{J}\right)^{k}\right],
$$

where ${ }_{2} \mathcal{F}_{1}$ is the hypergeometric function, while the calibration exponent was set to $k=6$. In general, there is no closed-form expression for ${ }_{2} \mathcal{F}_{1}$. However, the first derivative of $\Psi^{\mathrm{mag}}$ with respect to $I_{5} / J^{2}$, which is the one needed in the calculations, takes the simple explicit form

$$
\frac{\partial \Psi^{\mathrm{mag}}}{\partial\left(I_{5} / J^{2}\right)}=-\frac{\chi}{2 \mu_{0}(1+\chi)}\left[1+\left(\frac{\chi}{\mu_{0}(1+\chi) m^{\mathrm{s}}} \frac{\sqrt{I_{5}}}{J}\right)^{k}\right]^{-1 / k}
$$

For a more detailed discussion of this point, we refer the reader to Section 4.2.2 of Mukherjee et al. (2020) where $k \in \mathbb{N}^{+}$.

The magnetic saturation, $m^{\mathrm{s}}$, and the magnetic susceptibility, $\chi$, of the MRE are obtained in terms of the magnetic properties of the particles, i.e. the saturation magnetization $m_{\mathrm{p}}^{\mathrm{s}}$ and magnetic susceptibility $\chi_{\mathrm{p}}$, and their volume fraction $c$ as

and

$$
m^{\mathrm{s}}=c m_{\mathrm{p}}^{\mathrm{s}}
$$

$$
\chi=\frac{3 c\left(\mu_{\mathrm{p}}-\mu_{0}\right)}{(2+c) \mu_{0}+(1-c) \mu_{\mathrm{p}}} \quad \text { with } \quad \mu_{\mathrm{p}}=\left(1+\chi_{\mathrm{p}}\right) \mu_{0} .
$$

In turn, the magneto-mechanical coupling energy density has itself two contributions, i.e.,

with

$$
\Psi^{\text {couple }}\left(J, I_{5}, I_{6}\right)=\Psi_{6}\left(J, I_{6}\right)-\Psi_{5}\left(J, I_{5}\right)
$$

$$
\Psi_{i}\left(J, I_{i}\right)=\beta_{1} \frac{(1+\chi)\left(\mu_{0} m^{\mathrm{s}}\right)^{2}}{2 \mu_{0} \chi} \ln \left[1+\sum_{q=1}^{4} \frac{1}{c}\left(\frac{5}{4} \frac{\chi}{1+\chi}\right)^{q+1}\left(\frac{c}{\beta_{2}\left(\mu_{0} m^{\mathrm{s}}\right)^{2}} \frac{I_{i}}{J^{2}}\right)^{q}\right], \quad i=5,6
$$

The parameters $\beta_{1}$ and $\beta_{2}$ are given by

$$
\beta_{1}=\frac{16}{25} \frac{\mu_{0}^{2}(1+\chi)\left(v-(1+\chi) \mu_{0}\right)}{v \mu_{0} \chi\left(3(1+\chi) \mu_{0}-2 v\right)} \beta_{2}
$$


and

$$
\beta_{2}\left(G_{\mathrm{m}}^{*}, c\right)= \begin{cases}\alpha_{1}\left(G_{\mathrm{m}}^{*}\right)-\alpha_{2}\left(G_{\mathrm{m}}^{*}\right) \mathcal{L}\left[c \alpha_{3}\left(G_{\mathrm{m}}^{*}\right)\right], & \text { if } G_{\mathrm{m}}^{*} \leq 0.1 \\ 0.4055-0.5 c\left[1-0.67 \mathcal{L}\left(15 G_{\mathrm{m}}^{*}\right)\right] & \text { otherwise }\end{cases}
$$

with

$$
\begin{aligned}
v= & \mu_{0}+\frac{3 c\left(10+2 c+3 c^{2}\right)\left(\mu_{\mathrm{p}}-\mu_{0}\right) \mu_{0}^{2}}{5\left[(2+c) \mu_{0}+(1-c) \mu_{\mathrm{p}}\right]^{2}} \\
& +\frac{3 c(1-c)(5+3 c)\left(\mu_{\mathrm{p}}-\mu_{0}\right) \mu_{0} \mu_{\mathrm{p}}}{5\left[(2+c) \mu_{0}+(1-c) \mu_{\mathrm{p}}\right]^{2}}, \\
\alpha_{1}\left(G_{\mathrm{m}}^{*}\right)= & \exp \left[-0.029 \ln G_{\mathrm{m}}^{*}-0.982\right], \\
\alpha_{2}\left(G_{\mathrm{m}}^{*}\right)= & \exp \left[1.78 \mathcal{L}\left(-0.32 \ln G_{\mathrm{m}}^{*}\right)-1.78\right], \\
\alpha_{3}\left(G_{\mathrm{m}}^{*}\right)= & \exp \left[0.14-0.54 \ln G_{\mathrm{m}}^{*}\right] .
\end{aligned}
$$

Therein, the dimensionless parameter $G_{\mathrm{m}}^{*}$ is defined as

$$
G_{\mathrm{m}}^{*}=G_{\mathrm{m}} / G_{\mathrm{m}}^{\mathrm{ref}} \quad \text { with } \quad G_{\mathrm{m}}^{\mathrm{ref}}=1 \mathrm{MPa}
$$

whereas $\mathcal{L}$ denotes the Langevin function

$$
\mathcal{L}(x)=\frac{1}{\tanh (x)}-\frac{1}{x} .
$$

For completeness, we recall that $\beta_{1}$ allows to obtain the same initial magnetostriction as that resulting from the homogenized, implicit model of Lefèvre et al. (2017). On the other hand, $\beta_{2}$ is calibrated to numerical three-dimensional RVE calculations subjected to uniaxial magnetic fields and zero mechanical tractions.

Finally, the vacuum contribution is written as

$$
\Psi^{\mathrm{vac}}\left(J, I_{5}\right)=\frac{1}{2 \mu_{0} J} \mathbf{C}:(\mathbf{B} \otimes \mathbf{B}) .
$$

For the non-magnetic elastic materials, we simply use the same model as for the magneto-elastic ones but with particle volume fraction set to $c=0$. This readily leads to $\Psi^{\text {mag }}=\Psi^{\text {couple }}=0$ and to $\Psi^{\text {mech }}$ becoming just a NeoHookean solid, whereas $\Psi^{\mathrm{vac}}$ remains unchanged. Finally, as a result of the vanishing mechanical properties in air, $\Psi^{\mathrm{vac}}$ is the only term that survives in free space.

Remark 2. It should be pointed out here that the material parameters $\beta_{1}$ and $\beta_{2}$ (as well as the rest of the mechanical and magnetic parameters in this section) can be regarded as free parameters that may be used to calibrate a given experiment or numerical calculation where no information is given about the volume fraction of the particles. In this case, the proposed model becomes a purely phenomenological model, which, however, has a very simple structure and a rather small number of parameters that can be identified in a modular manner. For instance, one can identify the purely mechanical (such as the shear modulus) and magnetic parameters (such as $\chi$ and $m^{\text {s }}$ from independent mechanical and magnetic experiments. Subsequently, $\beta_{1}$ can be identified separately from $\beta_{2}$ from the initial quadratic response of the magnetostriction, while $\beta_{2}$ by probing the saturated magnetostriction.

\subsection{Material and geometric parameters}

In this section, we present the family of the analyzed geometries and describe in detail the corresponding material and geometric parameters that are varied in the following results sections.

Geometric parameters. With reference to Figure 2, we consider, in general, a thin film and an underlying substrate occupying the domains $\mathcal{B}^{\mathrm{f}}$ and $\mathcal{B}^{\mathrm{s}}$, respectively. We note further that in the present study, the substrate, unlike all previous studies in the literature, may also be magnetic and thus described by the fully-coupled magneto-mechanical model for MREs presented in the previous Section 3.1. This case is discussed in Sections 5 and 6.

We denote the total height of the body $h^{\mathrm{t}}$, the film thickness $h^{\mathrm{f}}$, thus implying that the height of the substrate is $h^{\mathrm{s}}=h^{\mathrm{t}}-h^{\mathrm{f}}$. The relevant parameter of the problem is the ratio $h^{\mathrm{f}} / h^{\mathrm{t}}$ since the layers extend to infinity in both 


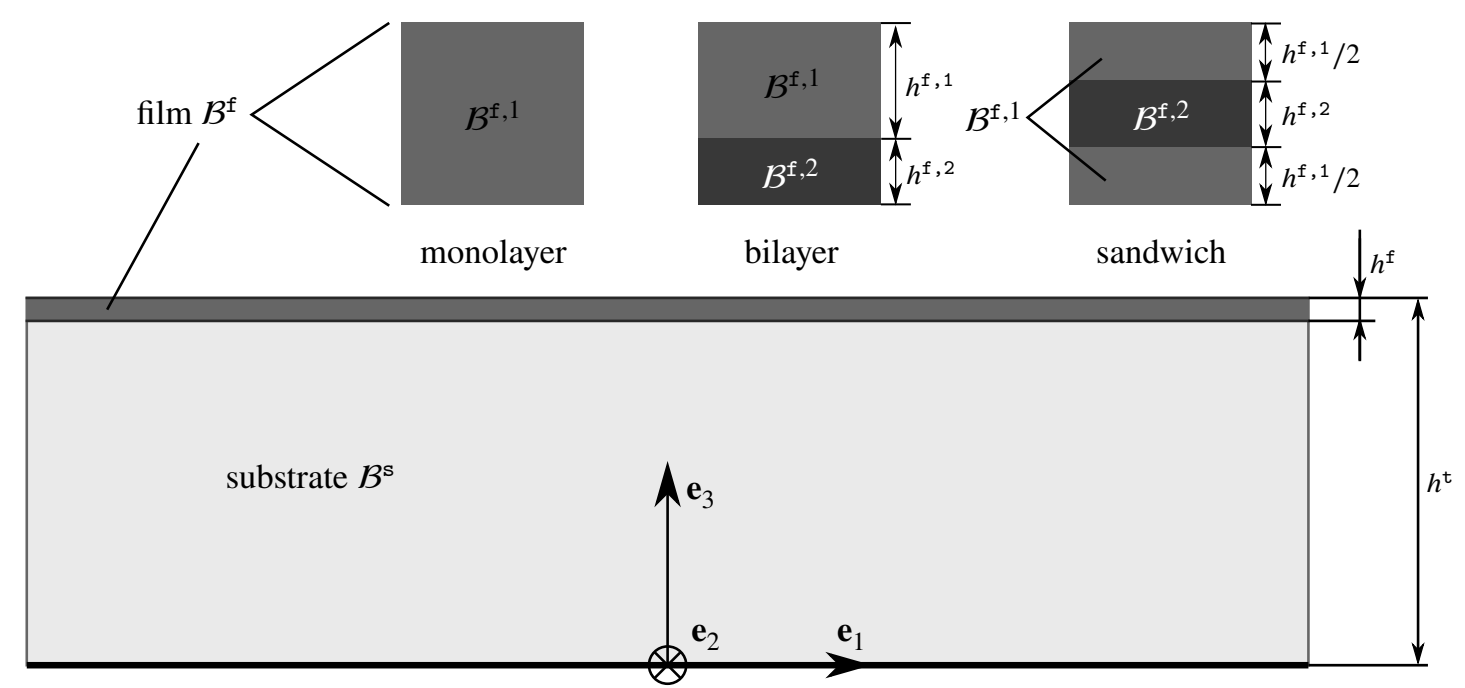

Figure 2: Sketch showing in two-dimensions the various film topologies considered in the present problem. We consider three topologies for the film; a monolayer with thickness $h^{\mathrm{f}}$, a bilayer and a sandwich with the last two cases allowing for a varying total thickness of the individual layers, $h^{\mathrm{f}, 1}$ and $h^{\mathrm{f}, 2}$ keeping the film thickness $h^{\mathrm{f}}$ fixed. The structure is fully defined by the ratio $h^{\mathrm{f}} / h^{\mathrm{t}}$ with $h^{\mathrm{t}}$ denoting the total height of the film and the substrate together.

$X_{1}-X_{2}$ directions. Nonetheless, for the sake of keeping the number of parameters tractable, we will focus only on thin films and set $h^{\mathrm{f}} / h^{\mathrm{t}}=1 / 50$, as shown in Table 1 . With regard to this point, it is important to mention that we have also analyzed different thicknesses of the MRE layer $h^{\mathrm{f}}$, while keeping the total height $h^{\mathrm{t}}$ constant. For ratios $h^{\mathrm{f}} / h^{\mathrm{t}} \leq 1 / 10$, we did not observe any significant effect as discussed in more detail in Appendix A.

Table 1: Geometric parameters

\begin{tabular}{lcc}
\hline Description & Symbol & Range \\
\hline $\begin{array}{l}\text { film height to total height } \\
\text { ratio }\end{array}$ & $h^{\mathrm{f}} / h^{\mathrm{t}}$ & $1 / 50$ \\
\hline $\begin{array}{l}\text { sub-film height ratio within } \\
\text { the film }\end{array}$ & $h^{\mathrm{f}, 2} / h^{\mathrm{f}, 1}$ & $0-10$ \\
\hline
\end{tabular}

Finally, in an effort to reduce the corresponding critical magnetic field required to attain the bifurcation, we consider two additional geometries, as shown in Figure 2, by splitting the top thin layer $\mathcal{B}^{\mathrm{f}}$ in two sub-films, $\mathcal{B}^{\mathrm{f}, 1}$ and $\mathcal{B}^{\mathrm{f}, 2}$, and with different topologies, such that $\mathcal{B}^{\mathrm{f}} \equiv \mathcal{B}^{\mathrm{f}, 1} \cup \mathcal{B}^{\mathrm{f}, 2}$. Again, in addition to the distribution of the two sub-films, i.e., their topology, the remaining relevant parameter is the ratio between their two corresponding heights, $h^{\mathrm{f}, 2} / h^{\mathrm{f}, 1}$, as defined in Table 1.

It is noted that throughout all examples, we employ free-space domains with a height of at least $100 h^{\mathrm{f}}$ above and below $\mathcal{B}$.

Material Parameters. The material parameters used in the subsequent result sections, attempt to examine the effect of the mechanical shear moduli ratios, such as $G^{\mathrm{s}} / G^{\mathrm{f}}$, as well as the magnetic response contrast, mainly determined by the particle volume fraction in each phase, e.g., $c^{\mathrm{s}} / c^{\mathrm{f}}$. The two sub-films as well as the substrate may be magnetic or not and are always chosen to be mechanically stiffer than the underlying substrate. The complete set and range of material parameters considered in the present study are summarized in Table 2.

Remark 3. The nonlinear saturation model used in equation (41) does not allow for a non-dimensionalization of the material ratios for the bifurcation response of the film-substrate block, as we will see later in the context of Fig. 6 . Moreover, it is important to notice that the magnetic bifurcation response is not only affected by the ratio of the shear moduli but also by the relative contrast between the magnetic properties of the layers as well as the contrast between the 
Table 2: Model parameters

\begin{tabular}{|c|c|c|}
\hline Description & Symbol & Range \\
\hline \multicolumn{3}{|c|}{ Magnetic properties } \\
\hline $\begin{array}{l}\text { magnetic permeability of } \\
\text { vacuum }\end{array}$ & $\mu_{0}\left(\mu \mathrm{N} \cdot \mathrm{A}^{-2}\right)$ & $0.4 \pi$ \\
\hline $\begin{array}{l}\text { magnetic susceptibility of } \\
\text { particles }\end{array}$ & $\chi_{\mathrm{p}}$ & 30 \\
\hline $\begin{array}{l}\text { saturation magnetization of } \\
\text { particles }\end{array}$ & $\mu_{0} m_{p}^{\mathrm{s}}(\mathrm{T})$ & 2.5 \\
\hline \multicolumn{3}{|c|}{ Mechanical properties } \\
\hline shear modulus of film & $G^{\mathrm{f}}(\mathrm{kPa})$ & $10-100$ \\
\hline $\begin{array}{l}\text { shear modulus of sub-film } \\
\text { layer } 1\end{array}$ & $G^{\mathrm{f}, 1}(\mathrm{kPa})$ & $10-15$ \\
\hline $\begin{array}{l}\text { shear modulus of sub-film } \\
\text { layer } 2\end{array}$ & $G^{\mathrm{f}, 2}(\mathrm{kPa})$ & $3-20$ \\
\hline shear modulus of substrate & $G^{\mathrm{s}}(\mathrm{kPa})$ & $1-5$ \\
\hline \multicolumn{3}{|c|}{ Particle volume fractions } \\
\hline $\begin{array}{l}\text { particle volume fraction in } \\
\text { the film }\end{array}$ & $c^{\mathrm{f}}$ & $0-0.2$ \\
\hline $\begin{array}{l}\text { particle volume fraction in } \\
\text { sub-film layers }\end{array}$ & $c^{\mathrm{f}, 1}, c^{\mathrm{f}, 2}$ & $0-0.2$ \\
\hline $\begin{array}{l}\text { particle volume fraction in } \\
\text { the substrate }\end{array}$ & $c^{\mathrm{s}}$ & $0-0.2$ \\
\hline
\end{tabular}

mechanical and magnetic parts of the energies. This implies that one needs to explore a very large range of parameters to have a complete picture of the effects. That is practically impossible. Nevertheless, we performed extensive studies which revealed a number of interesting effects but also far too many individual results to discuss each in detail. Thus, in the following sections, we focus on combinations of parameters that revealed novel and interesting phenomena.

Remark 4. It is important to clarify here that in the definition of the material model in Section 3.1 and particularly in equations (40) and (53), the underlying shear modulus is that of the polymeric matrix phase $G_{\mathrm{m}}$. Using then equation (40), one can readily recover the effective shear modulus ${ }^{6}$ as (Lopez-Pamies et al., 2013)

$$
G=\frac{G_{\mathrm{m}}}{(1-c)^{5 / 2}} \quad \text { or } \quad G_{\mathrm{m}}=(1-c)^{5 / 2} G
$$

Again, with reference to Table 2, we use $G \equiv G^{\mathrm{f}}$ to denote the effective shear modulus of the film, $G \equiv G^{\mathrm{s}}$ for the substrate and so on. In turn, we use the notation $G_{\mathrm{m}} \equiv G_{\mathrm{m}}^{\mathrm{f}}$ to denote the shear modulus of the matrix phase in the film, $G_{\mathrm{m}} \equiv G_{\mathrm{m}}^{\mathrm{s}}$ to denote the shear modulus of the matrix phase in the substrate. In the following studies, we may choose to work either with the effective shear modulus $G$ or the shear modulus of the underlying polymer matrix $G_{\mathrm{m}}$. The first option is of a more theoretical interest and relevant to the existing literature, whereas the second option allows to address experimental design questions similar to those in Psarra et al. (2017).

In the following sections, we discuss first the case of a single MRE layer on a passive substrate in Section 4. Subsequently, in Section 5, we add particles in the substrate while keeping a single MRE layer at the top. We finish by

\footnotetext{
${ }^{6}$ The expression for the effective shear modulus is valid in the incompressible limit. In our analysis, we use an sufficiently large second Lamé modulus and thus the result (56) holds to a very good approximation.
} 
generalizing our analysis to multi-layered structures in Section 6. Therein, we also attempt to respond to the question on how to minimize the critical magnetic field by varying the various material parameters and topologies at small pre-compression.

\section{Results: single MRE layer on a passive substrate}

This section deals with the single MRE monolayer topology in Figure 2 bonded to a passive, non-magnetic substrate. Thus, we use $G^{\mathrm{f}}$ and $c^{\mathrm{f}}$ to denote the overall shear modulus and the particle volume fraction in the MRE film. The set of parameters used in this example is given in Table 3. They represent the default values from which we deviate one-by-one in the parameter studies that follow. The values of these parameters correspond to the experimental ones

Table 3: Default parameters for the monolayer topology

\begin{tabular}{lccccc}
\hline Parameter & $G^{\mathrm{f}}(\mathrm{kPa})$ & $c^{\mathrm{f}}(-)$ & $G^{\mathrm{s}}(\mathrm{kPa})$ & $c^{\mathrm{s}}(-)$ & $h^{\mathrm{f}} / h^{\mathrm{t}}(-)$ \\
\hline Value & 10 & 0.2 & 3 & 0 & $1 / 50$ \\
\hline
\end{tabular}

discussed in Psarra et al. (2019) and are used as such in the following sections unless specified otherwise. The main quantities of interest are the critical magnetic field $b^{\mathrm{c}}$ and the current wave vector $\omega$, which is the observable one in an experimental setup, defined component-wise as

$$
\omega_{1}=\Omega_{1} / \lambda_{1} \text { and } \omega_{2}=\Omega_{2} / \lambda_{2}
$$

Moreover, for sufficiently thin films (with respect to the substrate) it is known that $\omega h^{\mathrm{f}}$ is the proper dimensionless quantity. In what follows, we will mainly discuss cases where the film is indeed sufficiently thin and thus employ $\|\omega\| h^{f}$ to characterize unstable modes. In most results that follow, we show bifurcation diagrams of the critical magnetic field $b^{\mathrm{c}}$ and the critical wave number amplitude $\|\omega\| h^{\mathrm{f}}$ as a function of the applied pre-compression $\lambda_{1}$ for given values of $\lambda_{2}$.

\subsection{Effect of biaxial pre-compression}

We begin with the analysis of a single MRE layer bonded to a passive substrate with material parameters as summarized in Table 3 that is subjected to a general in-plane biaxial pre-compression as described by the two pre-stretches $\lambda_{1} \leq 1$ and $\lambda_{2} \leq 1$ and a superposed transverse magnetic field. Figure 3 shows the critical magnetic field $b^{\mathrm{c}}$ and the dimensionless wave number $\|\omega\| h^{\mathrm{f}}$ as a function of the prescribed stretches $\left(\lambda_{1}, \lambda_{2}\right)$. The dashed lines correspond to fixed values of $\lambda_{2}$, while reducing $\lambda_{1}$ from $\lambda_{1}=1$ up to the point of a purely mechanical instability, that is when $b^{c}=0$. A first observation in Fig. 3a is the monotonic reduction of the critical magnetic field with increase of pre-compression (decrease of $\lambda_{1}$ ). More interestingly, we observe for the first time that the critical magnetic field generally decreases by increasing the pre-compression in the second direction (decrease of $\lambda_{2}$ ), i.e. biaxial pre-compression. For example, the entire bifurcation curve for $\lambda_{2}=0.85$ occurs at much lower magnetic fields for all $\lambda_{1}$ as compared to that for $\lambda_{2}=1$.

A special curve in Fig. 3 is the solid red line marking the locus of equi-biaxial applied strains. These states are of theoretical interest because the direction of the wave vector is undetermined for this load case (only the magnitude is determined) such that the actual pattern of the instability can only be determined by exploring the post-bifurcation regime (Chakrabarti et al., 2018). In particular, we observe in Fig. 3a that the $b^{c}$ curves exhibit a kink when crossing an equi-biaxial state. Similarly, in Fig. 3b, the equi-biaxial locus acts as a reflection for the amplitude of the wave numbers, leading to a sharp transition as one goes from $\lambda_{1}>\lambda_{2}$ to $\lambda_{1}<\lambda_{2}$. We emphasize here that, as indicated by the insets, by traversing the equi-biaxial locus, the actual wave vector changes direction: for $\lambda_{1}<\lambda_{2}$ we always obtain $\boldsymbol{\omega}=\left(\omega_{1}, 0\right)$, whereas the converse is true for $\lambda_{1}>\lambda_{2}$. This allows to plot both types of modes $\left(\omega_{1}, 0\right)$ and $\left(0, \omega_{2}\right)$ in terms of the amplitude $\|\omega\|$. The actual mode in action is indicated by the markers and the insets.

In order to have a more complete vision of the critical magnetic fields and the corresponding wave vectors, we show, in Figure 4, contour plots of the iso-lines of $b^{\mathrm{c}}$ and $\|\omega\| h^{\mathrm{f}}$, respectively, as functions of $\lambda_{1}$ and $\lambda_{2}$. Interestingly, the contours of $b^{\mathrm{c}}$ appear to be straight lines, again traversing the equi-biaxial case with a kink. While this is not exactly the case for $\|\omega\| h^{\mathrm{f}}$, curvature is still concentrated in the vicinity of the equi-biaxial line as can be seen in Figure $4 \mathrm{~b}$. 


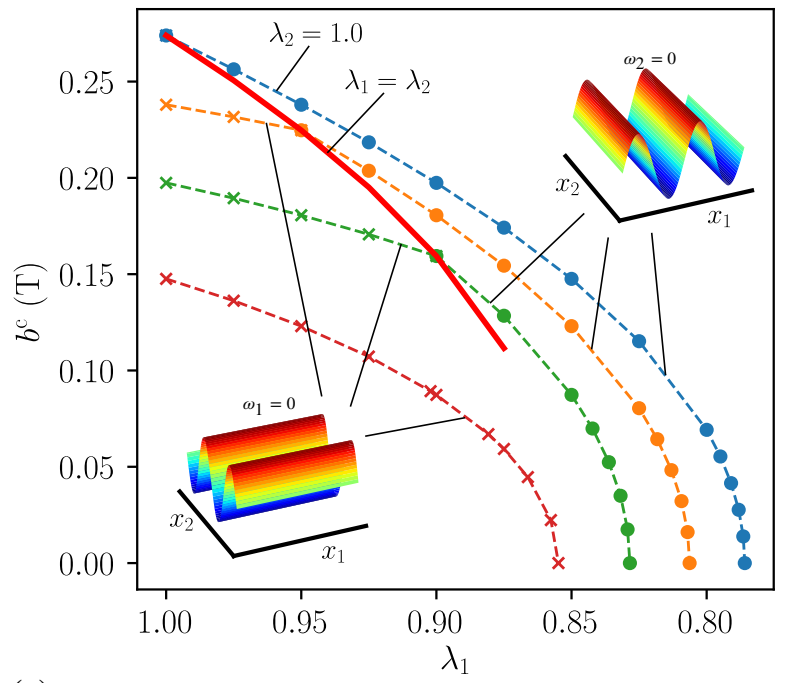

(a)

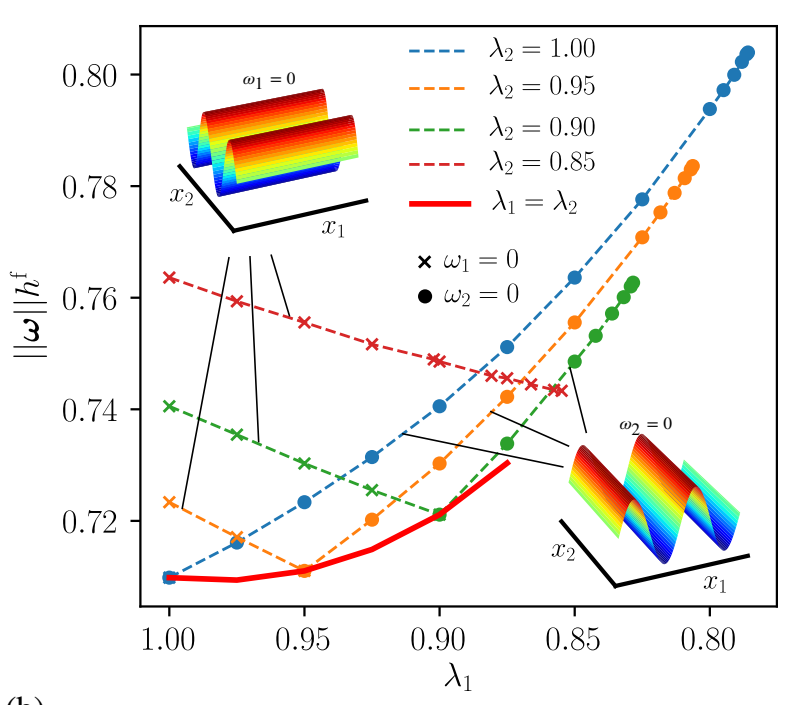

(b)

Figure 3: Effect of biaxial pre-compression for a MRE monolayer film on a passive substrate. (a) Bifurcation diagram of the critical magnetic field $b^{\mathrm{c}}$ and (b) critical wave number $\|\omega\| h^{\mathrm{f}}$ as a function of the stretch $\lambda_{1}$ for several values of the second stretch $\lambda_{2}$ (different colors). The solid (red) line depicts the locus of equi-biaxial states, i.e., $\lambda_{1}=\lambda_{2}$. As the bifurcation lines cross the solid line a sharp transition is observed. The symbols serve to denote a sinusoidal mode along $X_{1}\left(\omega_{2}=0\right)$ or $X_{2}\left(\omega_{1}=0\right)$ directions as explicitly sketched in the insets.

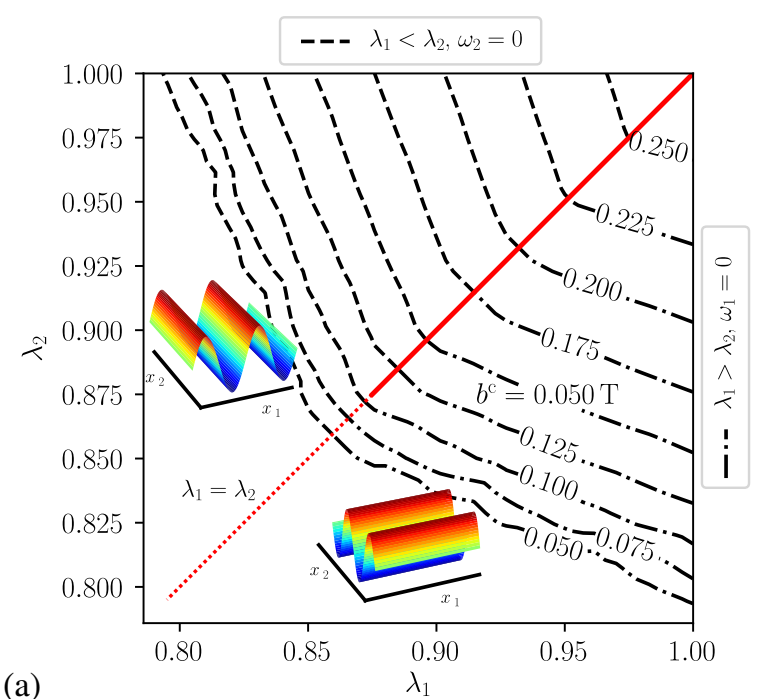

(a)

Figure 4: Effect of biaxial pre-compression for a MRE monolayer film on a passive substrate. Contour plots corresponding to (a) the bifurcation diagram of the critical magnetic field $b^{\mathrm{c}}$ and (b) the critical wave number $\|\omega\| h^{\mathrm{f}}$ as a function of the stretches $\lambda_{1} \lambda_{2}$. In both plots, the curvature of the contours is concentrated in the vicinity of the equi-biaxial locus (red line). Note that due to a limited resolution of the $\lambda_{1}-\lambda_{2}$ space, the local accuracy of the shown contours varies.

We conclude the discussion of this first representative case with Fig. 5, where we plot $b^{\mathrm{c}}$ over the components of $\omega h^{\mathrm{f}}$. This reveals clearly that in the special equi-biaxial case $\lambda_{1}=\lambda_{2}, \omega_{1} h^{\mathrm{f}}$ and $\omega_{2} h^{\mathrm{f}}$ satisfy the equation of a circle, wherein the amplitude $\|\omega\| h^{\mathrm{f}}$ remains constant, as shown by the dotted lines.

\subsection{Effect of the shear modulus of film}

In this section, we investigate the net effect of the shear modulus of the film $G^{\text {f }}$ upon the magneto-mechanical bifurcation response of the structure while keeping the ratio $G^{\mathrm{s}} / G^{\mathrm{f}}$ constant, i.e. $G^{\mathrm{s}}$ changes with $G^{\mathrm{f}}$. In turn, the 


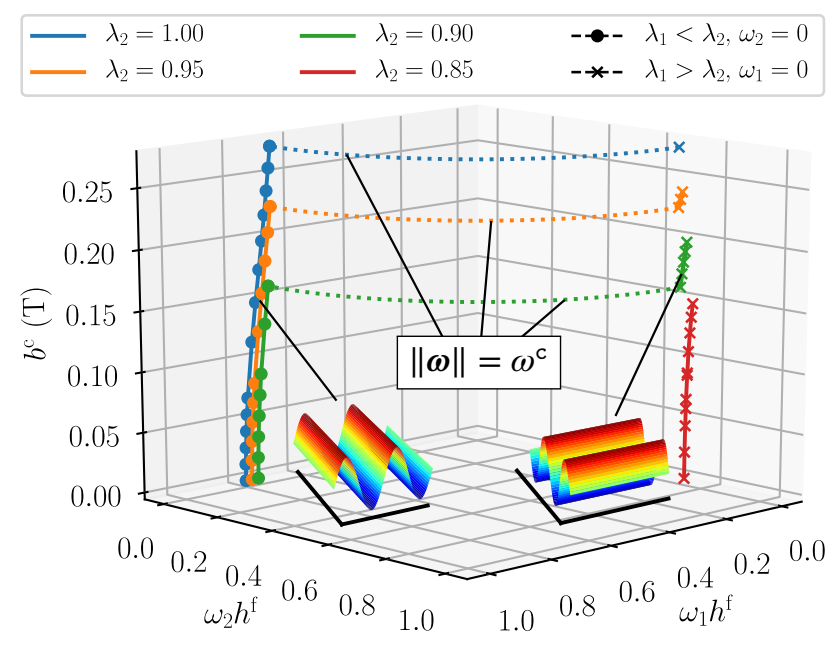

Figure 5: Effect of biaxial pre-compression for a MRE monolayer film on a passive substrate. A $3 \mathrm{~d}$ line plot of the critical magnetic field $b^{\mathrm{c}}$ over the normalized wave numbers $\omega_{1} h^{f}$ and $\omega_{2} h^{f}$. The dotted line corresponds to the equi-biaxial loading for which there is no unique value for $\omega$ but $\omega$ instead fulfills the equation of a circle with $\|\omega\|=\omega^{\mathrm{c}}$.

effect of biaxiality remains similar to the discussion done in Section 4.1 and thus we fix for simplicity $\lambda_{2}=1$. The values of the shear moduli considered are given as ${ }^{7}$

$$
G^{\mathrm{f}} \in\left\{10,50,100,200,500,10^{3}, 5 \times 10^{3}\right\} \mathrm{kPa} \quad \text { and } \quad G^{\mathrm{s}} / G^{\mathrm{f}}=0.3,
$$

while keeping $c^{\mathrm{f}}=0.2$. This implies that the magnetic properties of the MRE film do not change with $G^{\mathrm{f}}$ but $G^{\mathrm{s}}$ changes such that $G^{\mathrm{s}} / G^{\mathrm{f}}$ is maintained. This leads to an identical critical bifurcation response in the absence of a magnetic field, as easily observed in Fig. 6a, where all curves coincide for $b^{c}=0$. By contrast, in the same figure, we observe that the $b^{\mathrm{c}}$ is strongly dependent upon the value of $G^{\mathrm{f}}$, an effect that was missed in earlier studies (see for instance Danas and Triantafyllidis (2014) which studied values in the order of $1 \mathrm{MPa}$ ). Interestingly, we find that the critical magnetic field behaves as $b^{\mathrm{c}} \sim \sqrt{G^{\mathrm{f}}}$ for low values of $G^{\mathrm{f}}$, as clearly shown by the inset in the same figure, but deviates for larger ones. The explanation derives from the fact that for large $G^{\mathrm{f}}$ the saturation response of the MRE film plays an important role leading to this vertical asymptotic response of $b^{\mathrm{c}}$. As can be seen from the inset in Fig. 6b, the critical magnetization curves $m^{\mathrm{c}}$ for $G^{\mathrm{f}} \geq 200 \mathrm{kPa}$ reach the saturation magnetization $m^{\mathrm{s}}$ at finite precompressions $\lambda_{1}<1$. As a consequence, no magneto-mechanical bifurcations can be obtained past that saturation point. This leads to the steep increase of $b^{c}$ in part (a) of this figure. Moreover, this nonlinear magnetization response breaks down the scaling of $b^{\mathrm{c}}$ with $G^{\mathrm{f}}$ (see inset in Fig. 6a). The saturating response does not affect significantly $b^{\mathrm{c}}$ for lower $G^{\mathrm{f}}<200 \mathrm{kPa}$ since the bifurcation actually occurs at much lower magnetic fields.

On the other hand, in Fig. $6 \mathrm{~b}$, the critical wave vector amplitude is fairly insensitive to the value of $G^{\mathrm{f}}$ alone, while it is mostly affected by the ratio $G^{\mathrm{s}} / G^{\mathrm{f}}$, as we will see in the next Section 4.3.

We note that the strong effect of $G^{\mathrm{f}}$ upon the bifurcation response of the film-substrate structure has significant implications on experiments and on potential applications as a result of the very narrow range of pre-compressions that affects $b^{\mathrm{c}}$ for large $G^{\mathrm{f}}>500 \mathrm{kPa}$. In turn, for softer MRE films, the bifurcation response spans the entire range of pre-compressions leading to an important effect of $\lambda_{1}$ upon $b^{\mathrm{c}}$.

We close this section by recalling that the work of Psarra et al. (2017) used a MRE film of $G^{\mathrm{f}}=10 \mathrm{kPa}$ and very similar values were obtained experimentally for the bifurcation response. This makes the present study highly relevant for actual experimental design, even though no post-bifurcation response is resolved. This observation is mainly due to the super-critical character of the post-bifurcation response in the present cases as discussed extensively in Psarra et al. (2019).

\footnotetext{
${ }^{7}$ Here we go beyond the range for $G^{\mathrm{f}}$ and $G^{\mathrm{s}}$ in Table 2 to reveal the full range of the effect of their absolute value.
} 


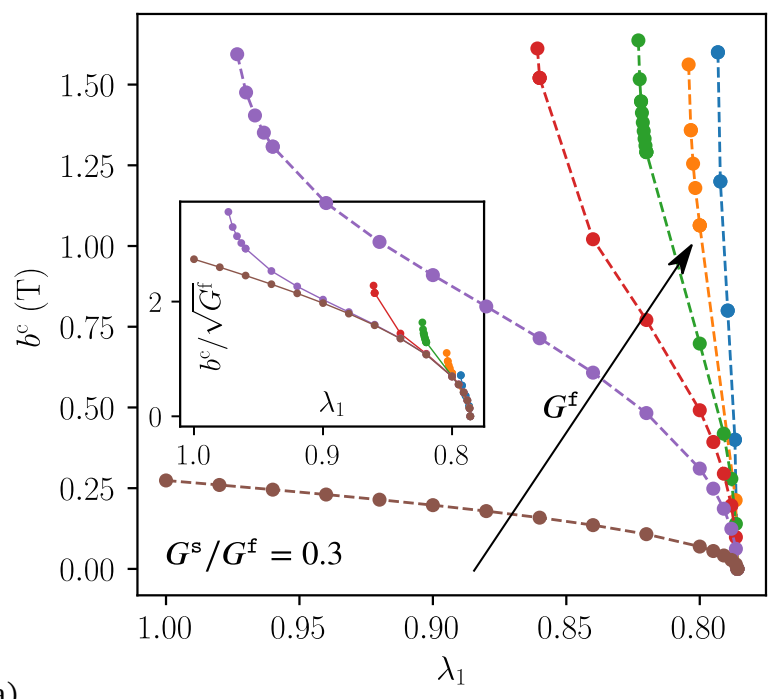

(a)

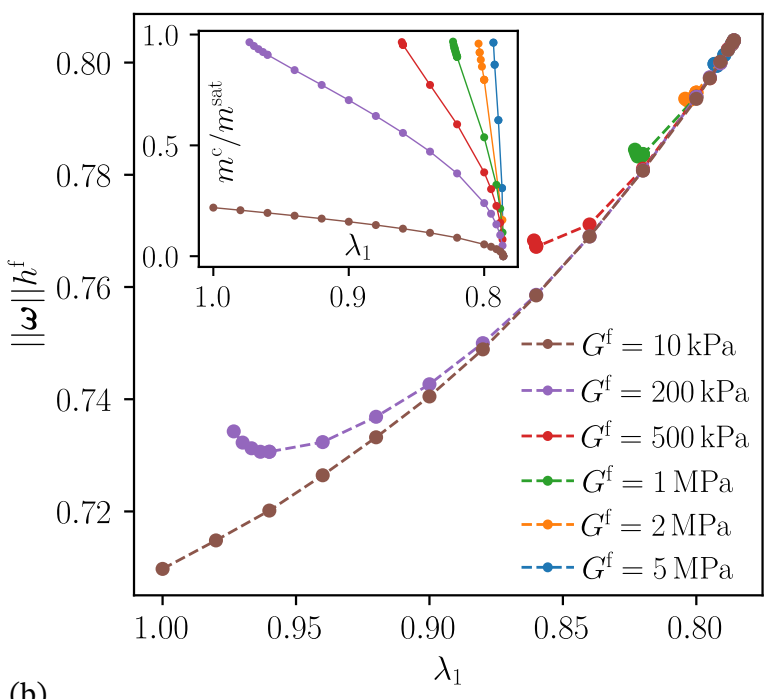

(b)

Figure 6: Effect of the shear modulus on the bifurcation behavior of a MRE monolayer film layer bonded on a passive substrate. While varying $G^{\mathrm{f}}$, the ratio of moduli and the particle volume fraction of the MRE film are set to $G^{\mathrm{s}} / G^{\mathrm{f}}=0.3$ and $c^{\mathrm{f}}=0.2$, respectively. Bifurcation diagrams of (a) the critical magnetic field $b^{\mathrm{c}}$ and (b) the critical wave number $\|\omega\| h^{\mathrm{f}}$. The inset in (a) shows the critical field $b^{\mathrm{c}}$ scaled with $1 / \sqrt{G^{\mathrm{f}}}$. The inset in (b) depicts the normalized critical magnetization $\mathrm{m}^{\mathrm{c}} / \mathrm{m}^{\mathrm{s}}$.

\subsection{Effect of substrate-to-film shear modulus ratio}

In this section, we investigate the effect of mechanical stiffness ratio $G^{\mathrm{s}} / G^{\mathrm{f}}$ upon the magneto-mechanical bifurcation response keeping $G^{\mathrm{s}}$ fixed. For this, we consider the following values

$$
G^{\mathrm{s}} / G^{\mathrm{f}} \in\{0.1,0.2,0.3,0.4\} \quad \text { with } \quad G^{\mathrm{s}}=3 \mathrm{kPa},
$$

keeping with $c^{\mathrm{f}}=0.2$ (i.e. the magnetic properties of the film do not change) and $\lambda_{2}=1$. The rest of the values are the ones defined in Table 2. Also, we note that $G^{\mathrm{s}} / G^{\mathrm{f}}$ remains sufficiently below the value of 0.575 beyond which the mechanical response tends to become imperfection sensitive (Cai and $\mathrm{Fu}, 1999$ ) and thus the practicality of the present bifurcation analysis gradually breaks down since the post bifurcation (real) response and pattern can be substantially different that that predicted by the bifurcation analysis discussed in Section 2.2. In Fig. 7a, we observe as expected that over a wide range of pre-compressions, a lower ratio $G^{\mathrm{s}} / G^{\mathrm{f}}$ yields a lower critical magnetic field $b^{\mathrm{c}}$. This is easily explained by observing that the purely mechanical instability (i.e., at $b^{\mathrm{c}}=0$ ) occurs at lower pre-compressions (i.e. larger $\lambda_{1}$ ) for smaller $G^{\mathrm{s}} / G^{\mathrm{f}}$. In the purely mechanical case, the ratio of shear moduli alone determines the bifurcation point in the $\lambda_{1}$ axis. By contrast, at smaller pre-compressions, i.e. values of $\lambda_{1}>0.95$, the critical curves inter-cross leading to a lower critical magnetic field $b^{\mathrm{c}}$ for larger $G^{\mathrm{s}} / G^{\mathrm{f}}$. Furthermore, in Fig. $7 \mathrm{~b}$ we observe a significant effect of the shear modulus ratio on the wave numbers $\|\omega\| h^{\mathrm{f}}$. In particular, a higher $G^{\mathrm{s}} / G^{\mathrm{f}}$ leads to a larger $\|\omega\| h^{\mathrm{f}}$. In practice, this implies a larger number of wrinkles with increasing shear moduli ratio. Nonetheless, the range of changes of the wave number for a given moduli ratio is quite limited.

\subsection{Effect of magnetic properties via the particle volume fraction in the film}

In this section, we examine the effect of the particle volume fraction in the film, and consequently of its magnetic properties. For simplicity, we set a value for the shear modulus of the underlying matrix phase of the film, $G_{\mathrm{m}}^{\mathrm{f}}$ and vary $c^{\mathrm{f}}$. Thus, the key parameters are

$$
c^{\mathrm{f}} \in\{0.1,0.15,0.2,0.25\}, \quad G_{\mathrm{m}}^{\mathrm{f}}=5.72 \mathrm{kPa} .
$$

This value for $G_{\mathrm{m}}^{\mathrm{f}}$ is obtained by use of relation (56) and motivated by the experimental study of Psarra et al. (2019), where the film modulus was found to be $G^{\mathrm{f}}=10 \mathrm{kPa}$ for $c^{\mathrm{f}}=0.2$. Again, we keep $G^{\mathrm{s}}=3 \mathrm{kPa}$ and $\lambda_{2}=1$. 


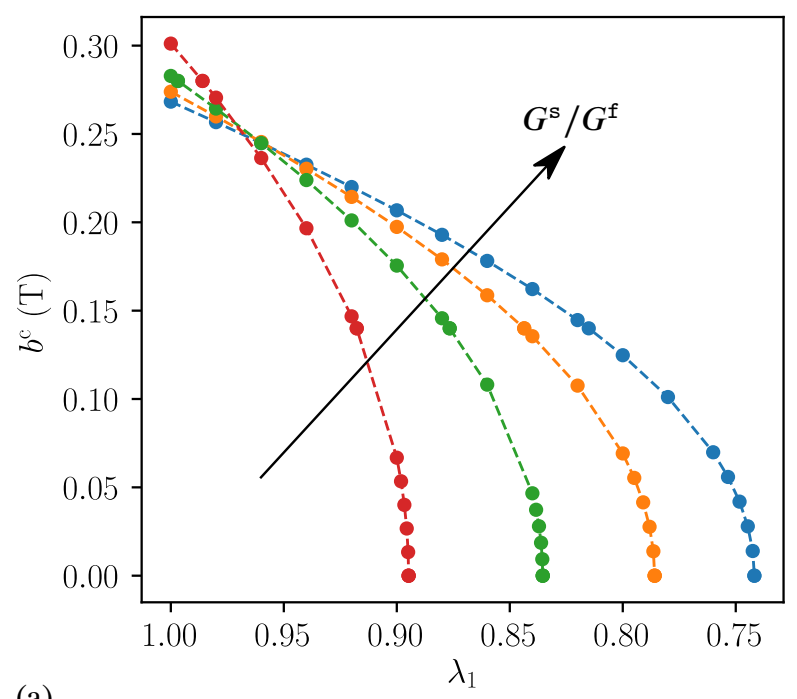

(a)

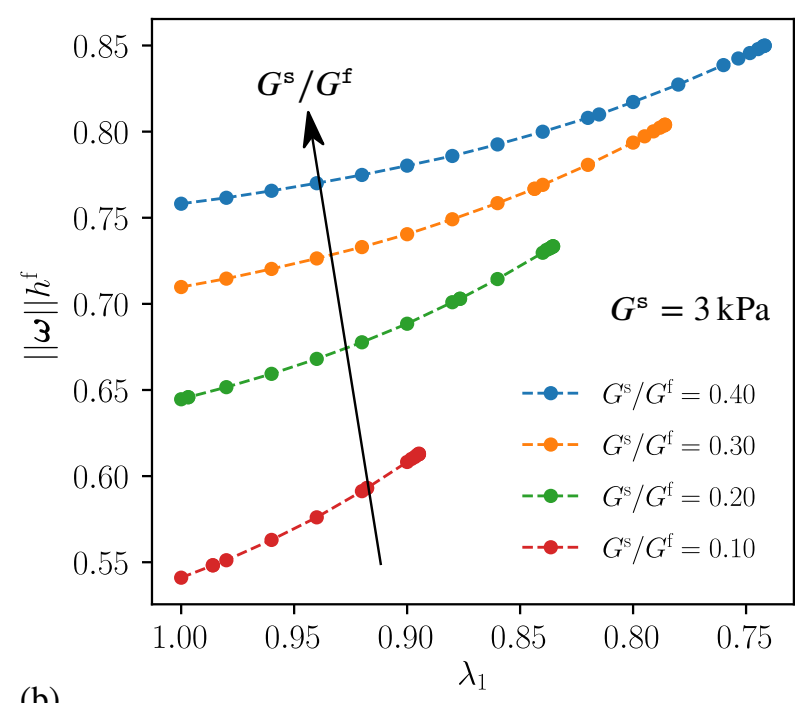

(b)

Figure 7: Effect of the substrate-to-film shear modulus ratio on the bifurcation behavior of a MRE monolayer film layer bonded on a passive substrate. Bifurcation diagrams of (a) the critical magnetic field $b^{\mathrm{c}}$ and (b) the critical wave number $\|\omega\| h^{\mathrm{f}}$. The ratio of moduli has a significant effect on both the critical field $b^{\mathrm{c}}$ and the corresponding wave numbers $\|\omega\| h^{\mathrm{f}}$.

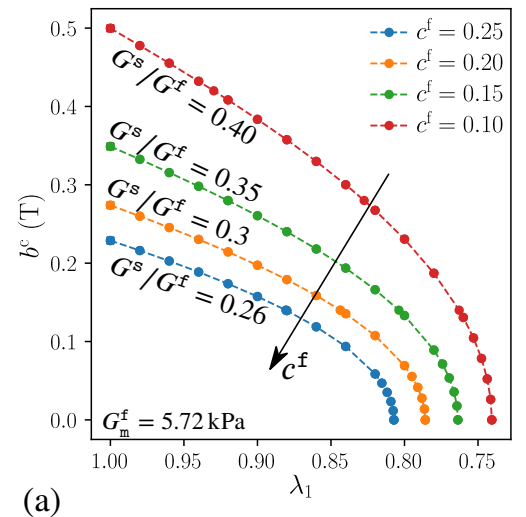

(a)

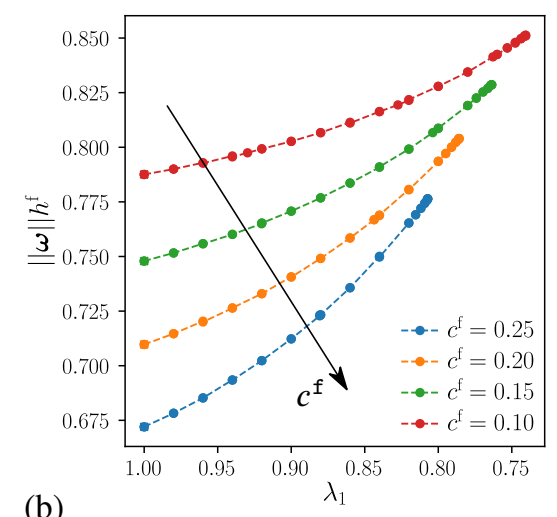

(b)

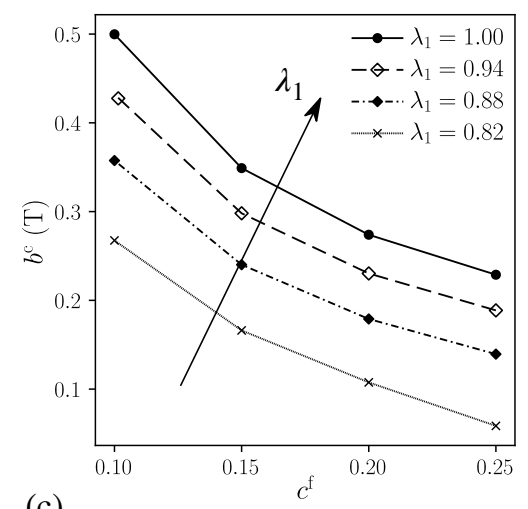

(c)

Figure 8: Effect of the particle volume fraction in the MRE layer on the bifurcation behavior. Bifurcation diagrams of (a) the critical magnetic field $b^{\mathrm{c}}$ and (b) the critical wave number $\|\omega\| h^{\mathrm{f}}$. (c) shows a cross-plot of $b^{\mathrm{c}}$ as a function of the particle volume fraction $c^{\mathrm{f}}$ for selected stretch states $\lambda_{1}$.

Figure 8a shows a significant effect of the particle volume fraction $c^{f}$ upon the critical magnetic field $b^{c}$. Specifically, increase of $c^{\mathrm{f}}$ leads to an overall decrease of both the critical magnetic field $b^{\mathrm{c}}$ and the critical pre-compression at $b^{\mathrm{c}}=0$. This is explained by the fact that increase of $c^{\mathrm{f}}$ leads to increase of the magnetic susceptibility $\chi$ of the MRE film and hence bifurcation appears at lower magnetic fields. The mechanical stiffening effect resulting from the increase of $c^{\mathrm{f}}$ is in turn counteracted by the simultaneous decrease of $G^{\mathrm{s}} / G^{\mathrm{f}}$. The latter leads to a lower critical pre-compression in the purely mechanical case (i.e. $b^{\mathrm{c}}=0$ ).

The corresponding results for the wave number in Fig. $8 \mathrm{~b}$ are qualitatively similar to the ones presented in the previous sections. In particular, increase of $c^{f}$ leads to lower wave numbers (i.e. fewer wrinkles), whereas the range of changes of the wave number for a given particle volume fraction is quite limited.

Finally, in Fig. 8c, we show $b^{\mathrm{c}}$ as a function of the particle volume fraction in the film $c^{\mathrm{f}}$ for a fixed $\lambda_{1}$. The main result here is clear reduction of $b^{\mathrm{c}}$ with the increase of $c^{\mathrm{f}}$. As expected, the lowest values for $b^{\mathrm{c}}$ occur for the highest pre-compression $\lambda_{1}$. 


\section{Results: a single MRE layer on a MRE substrate}

In this section we first highlight the effect of magnetic particles in the substrate by varying their volume fraction $c^{\mathrm{s}}$ for uniaxial pre-compression, i.e., $\lambda_{1} \leq 1$ and $\lambda_{2}=1$. Subsequently, we present two selected cases that explore a large range of biaxial pre-compression loading states with $\lambda_{1} \leq 1$ and $\lambda_{2} \leq 1$.

\subsection{Effect of particle volume fraction in the substrate}

In this section, we add magnetic particles both in the substrate and the film at different volume fractions $c^{\mathrm{s}}$ and $c^{f}$, respectively. The addition of particles evidently changes both the mechanical and magnetic properties of the film and substrate materials in a nonlinear manner (see studies of Lopez-Pamies et al. (2013); Keip and Rambausek (2016); Danas (2017); Lefèvre et al. (2017)). Nonetheless, in order to keep the analysis tractable, we decide to vary only $c^{\text {s }}$ and fix the rest of the material properties as

$$
c^{\mathrm{s}} \in\{0,0.05,0.1,0.15,0.2\}, \quad c^{\mathrm{f}}=0.1, \quad G^{\mathrm{f}}=10 \mathrm{kPa}, \quad G^{\mathrm{s}}=3 \mathrm{kPa},
$$

such that $G^{\mathrm{s}} / G^{\mathrm{f}}=0.3$ independently of the variation of $c^{\mathrm{s}}$. For this to be achieved, one needs to change the shear modulus of the underlying polymer matrix in the substrate $G_{\mathrm{m}}^{\mathrm{s}}$ by using equation (56) accordingly. In practice, that can be achieved by changing the ingredients allowing the polymerization of the matrix (see for instance Bodelot et al. (2018)).

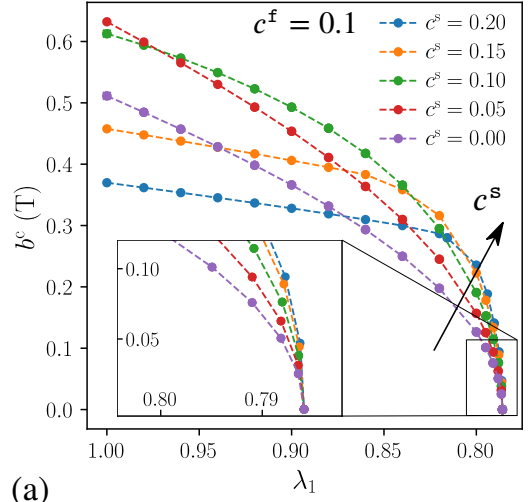

(a)

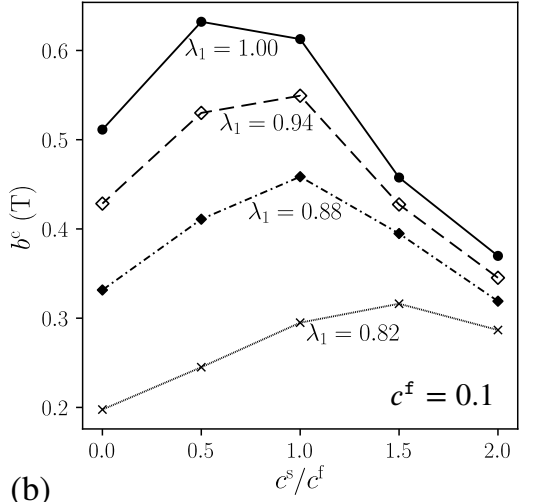

(b)

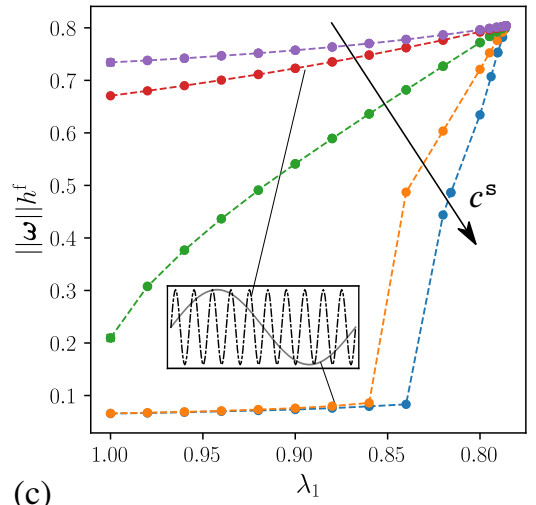

(c)

Figure 9: Effect of the substrate particle volume fraction for a MRE layer on a MRE substrate. While varying $c^{\mathbf{s}}$, the parameters $G^{\mathbf{s}}=3 \mathrm{kPa}$, $G^{\mathrm{f}}=10 \mathrm{kPa}$ and $c^{\mathrm{f}}=0.1$ are fixed. Bifurcation diagrams for the critical magnetic field $b^{\mathrm{c}}$ (a) and the critical wave vector amplitude $\|\omega\| h^{\mathrm{f}}$ (c). Subplot (b) depicts a cross-plot of $b^{\mathrm{c}}$ as a function of the ratio of volume fraction $c^{\mathrm{s}} / c^{\mathrm{f}}$ for several values of $\lambda_{1}$.

We commence our discussion with Fig. 9a and particularly the purely mechanical bifurcation point corresponding to $b^{\mathrm{c}}=0$ and $\lambda_{1} \approx 0.78$. Departing from that point, the increase of the magnetic field (for a fixed $\lambda_{1}$ ) stiffens the substrate material. The stiffening effect is more siginificant with the increase of the particle concentration $c^{\mathbf{s}}$ in the substrate due to its larger volume (Keip and Rambausek, 2016; Danas, 2017). Note, in turn, that the purple curve (see also inset) corresponding to $c^{\mathrm{s}}=0$ leads to the lowest critical magnetic field $b^{\mathrm{c}}$ near the purely mechanical bifurcation region. Nevertheless, for lower pre-compressions (i.e. larger $\lambda_{1}$ ) and larger magnetic fields, the substrate with the highest volume fraction $c^{\mathrm{s}}=0.2$ inter-crosses the rest of the curves, leading to the lowest $b^{\mathrm{c}}$ among all cases studied here. This effect is shown clearly in Fig. 9b, where for a given value of $\lambda_{1}$, we show the cross-plot of $b^{\mathrm{c}}$ as a function of the ratio $c^{\mathrm{s}} / c^{\mathrm{f}}$ with $c^{\mathrm{f}}=0.1$. Therein, we observe a non-monotonic behavior of $b^{\mathrm{c}}$ at lower pre-compressions exhibiting a maximum that roughly lies near $c^{\mathrm{s}} / c^{\mathrm{f}} \sim 1$. This implies that the more magnetic the substrate is, the more unstable the structure becomes - especially at lower pre-compressions.

In turn, Fig. 9c shows the corresponding wave numbers $\|\omega\| h^{\mathrm{f}}$ as a function of $\lambda_{1}$. For large $c^{\mathrm{s}}$ where the substrate is more magnetic than the film, a very low wave number $\|\omega\| h^{\mathrm{f}}<0.1$ is obtained implying a rather long-wavelength wrinkling mode. By contrast, when the $c^{\mathrm{s}} / c^{\mathrm{f}}<1$, the wave number is very high implying significant number of wrinkles in the structure. This difference is shown in the inset sinusoidal sketch for two such representative points indicated directly on the graph. An additional impressive result is the sharp increase of $\|\omega\| h^{\mathrm{f}}$ for the cases $c^{\mathrm{s}} / c^{\mathrm{f}} \geq 1$. 
This simply suggests that, in practice, a small additional pre-compression can lead to a very sharp increase of the number of wrinkles and a drop of $b^{c}$ in the structure and thus a pattern switching with marginal energy changes. Such an effect requires further studies in the post-bifurcation regime to test its validity in practical cases but even so it shows a promising mechanism for pattern switching.

\subsection{Effect of biaxial pre-compression}

In connection with the previous section, we investigate the effect of biaxial pre-compression by varying $\lambda_{2} \leq 1$ for two cases; one where $c^{\mathrm{s}}=c^{\mathrm{f}}=0.2$ in Fig. 10 and a second with $c^{\mathrm{s}}=0.2$ and $c^{\mathrm{f}}=0.1$ in Fig. 11 . This allows to show a qualitative transition of the bifurcation response obtained by considering a substrate that is more magnetic than the film.

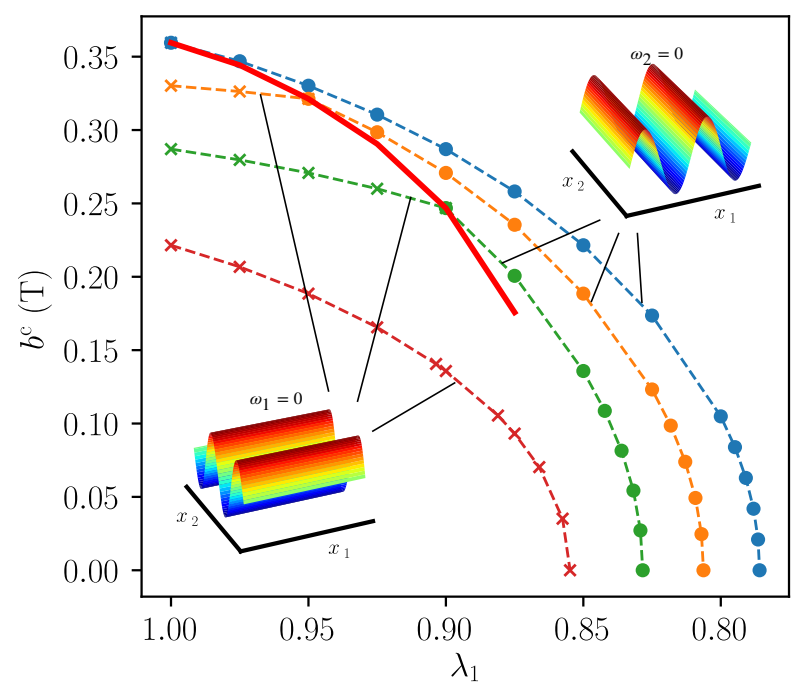

(a)

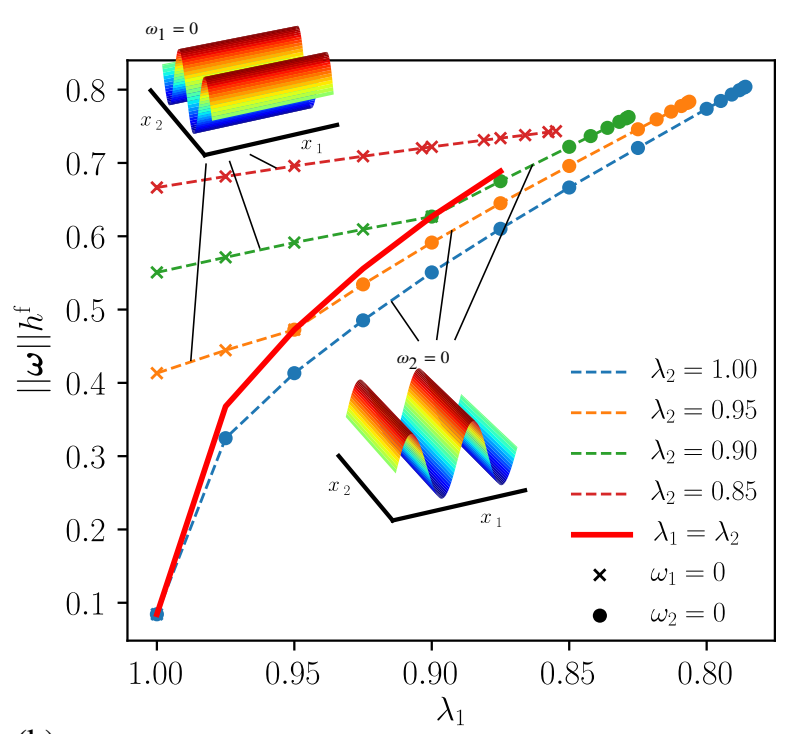

(b)

Figure 10: Effect of biaxial precompression of a MRE monolayer film bonded to an equally magnetic but mechanically softer MRE substrate. The key parameters are $c^{\mathrm{s}}=c^{\mathrm{f}}=0.2, G^{\mathrm{f}}=10 \mathrm{kPa}$ and $G^{\mathrm{s}}=3 \mathrm{kPa}$. (a) Bifurcation diagram of the critical magnetic field $b^{\mathrm{c}}$ and (b) the critical wave number amplitude $\|\omega\| h^{f}$ as a function of the stretch $\lambda_{1}$ for several values for $\lambda_{2}$. As the bifurcation lines cross the equi-biaxial locus (solid red line) one observes a kink in the graphs leading also to a change of direction of the critical modes as indicated by the insets and the markers.

Specifically, Fig. 10a, corresponding to $c^{\mathrm{s}}=c^{\mathrm{f}}=0.2$, shows a reduction of the critical magnetic field $b^{\mathrm{c}}$ with the increase of the biaxial pre-compression, i.e., decrease of $\lambda_{2}$. This decrease is smooth and shows a concave character similar to the one for the passive substrate, discussed in Fig. 3. It should be mentioned that the as one crosses the equi-biaxial pre-compression regime (denoted with a thick continuous line on the graph), $b^{c}$ curves show a sharp transition and a change of the direction of the wrinkling mode. This sharp transition is more clear in Fig. 10b for the corresponding wave number $\|\omega\| h^{f}$. As already noted in the context of Fig. 5, for $\lambda_{1}=\lambda_{2}$, the amplitude of the wave number is defined but not the exact ratio. For that a post-bifurcation analysis is required and is left for a future study. Finally, we note that the change of the wave number and hence the number of potential wrinkles in the specimen vary more significantly for $\lambda_{2}=1$ as a function of $\lambda_{1}$ than for $\lambda_{2}=0.85$.

By contrast, as shown in Fig. 11, as one decreases the particle volume fraction of the film to $c^{\mathrm{f}}=0.1$, while keeping that of the substrate to $c^{\mathrm{s}}=0.2$ (i.e. $c^{\mathrm{s}} / c^{\mathrm{f}}=2$ ), a qualitative difference is observed both for the critical magnetic field $b^{\mathrm{c}}$ and the corresponding wave number $\|\omega\| h^{\mathrm{f}}$. In particular, from Fig. 11b, one may extract two regimes; regime I and II delimiting the long and short wavelength surface pattern response, respectively. The boundary separating those two regimes is point-by-point transferred also to Fig. 11a showing the critical magnetic field $b^{\mathrm{c}}$.

As can be directly observed in Fig. 11a, the transition between regime I (long wavelengths) and II (short wavelengths) only occurs for critical fields $b^{\mathrm{c}} \sim 0.3 \mathrm{~T}$, while it is not present for significant pre-compression such as 

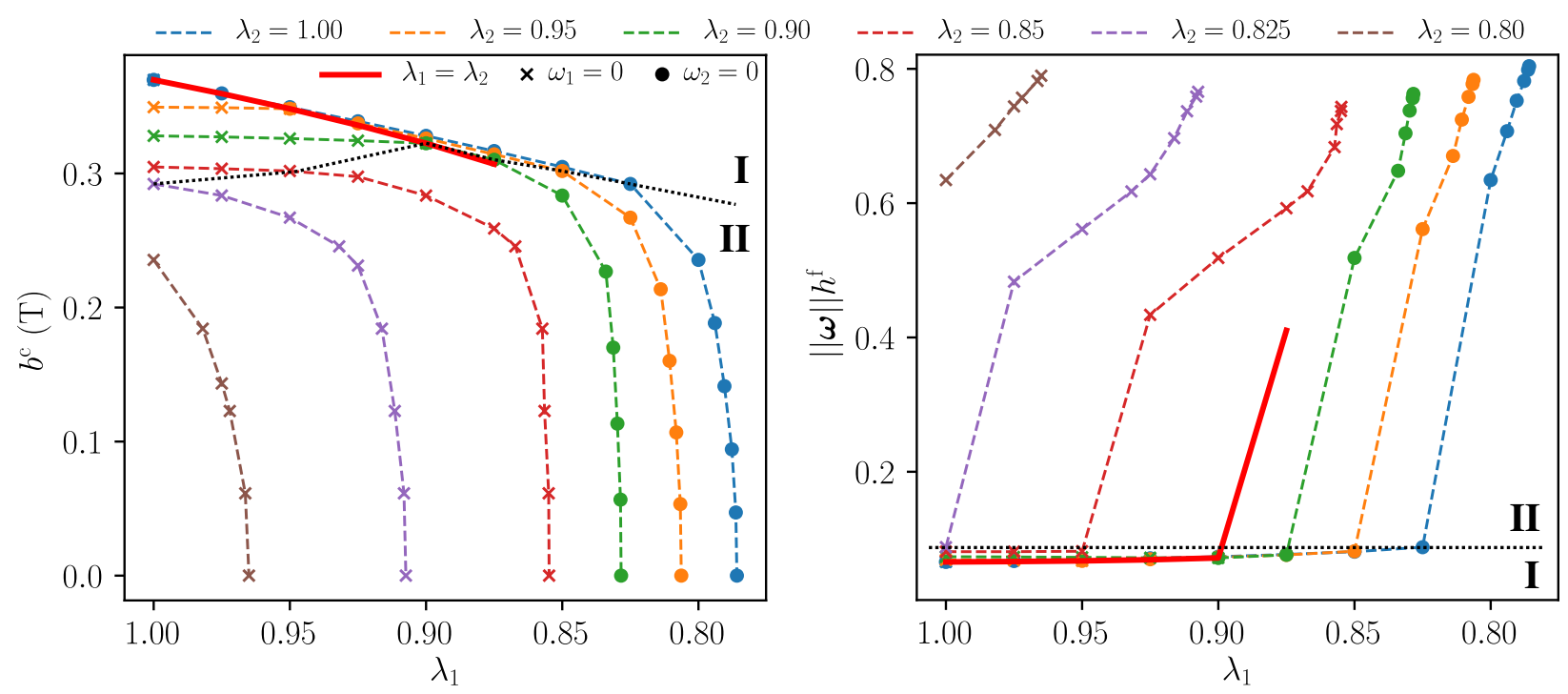

Figure 11: Effect of biaxial precompression of a MRE monolayer film bonded to a magnetically dominant but mechanically softer MRE substrate. The key parameters are $c^{\mathrm{s}}=0.2=2 c^{\mathrm{f}}, G^{\mathrm{f}}=10 \mathrm{kPa}$ and $G^{\mathrm{s}}=3 \mathrm{kPa}$. (a) Bifurcation diagram of the critical magnetic field $b^{\mathrm{c}}$ and (b) the critical wave vector amplitude $\|\boldsymbol{\omega}\| h^{\mathrm{f}}$ as a function of the stretch $\lambda_{1}$ for several values for $\lambda_{2}$. The roman numbers $\mathbf{I}$ and $\mathbf{I I}$ denote a magnetically governed long-wavelength and a mechanically governed long-to-short wave-length regime. The markers for $\omega_{1}=0$ and $\omega_{2}=0$ indicate the respective direction of the critical mode.

$\lambda_{2}=0.8^{8}$. Specifically, in regime $\mathbf{I}$, we observe a very low sensitivity of $b^{\mathrm{c}}$ to $\lambda_{1}$ leading to a response that is mainly governed by the magnetic properties of the film and substrate. Note that a slight kink is still found for the bifurcation curves crossing the equi-biaxial locus. In turn, regime II is characterized by an extremely sharp increase of $b^{\mathrm{c}}$ starting from the purely mechanical bifurcation point for a given biaxial $\lambda_{1}-\lambda_{2}$ pair. This sharp increase of $b^{\mathrm{c}}$ implies that, in an actual experiment operated near that regime, it is very difficult to obtain wrinkling at low magnetic fields since one would need an extremely precise control of the pre-compression device in this case. Thus, in practice it might be favorable to operate an experiment near the transition from $\mathbf{I}$ to II where the sensitivity of $b^{\mathrm{c}}$ with respect to the pre-compression is important. In this context we remark that the curves for $\lambda_{2} \in[0.825,0.9]$ exhibit the most tractable behavior.

Figure 11b shows a number of interesting features for the corresponding critical wave number $\|\omega\| h^{\mathrm{f}}$. First of all, regime I corresponds to wave numbers $\|\omega\| h^{\mathrm{f}} \sim 0.05$, which translates to wavelengths in the order of $100 h^{\mathrm{f}}$, i.e. spanning the size of a typical specimen (Psarra et al., 2017). This regime is the magnetically dominated regime in connection with Fig. 11a. The transition of $\|\omega\| h^{\mathrm{f}}$ to regime II is extremely sharp (almost jumps) thus offering the possibility for pattern switching with only minor changes in the applied pre-compression $\lambda_{1}$. The maximum values of $\|\omega\| h^{\mathrm{f}}$ corresponding to the purely mechanical bifurcation response reach values as high as $\|\omega\| h^{\mathrm{f}}=0.8$. This implies, in turn, that regime II is dominated by the mechanical properties of the film/substrate block and more specifically by the mechanical stiffness ratio $G^{\mathrm{s}} / G^{\mathrm{f}}$. With the application of the magnetic field, $\|\omega\| h^{\mathrm{f}}$ evolves from the short wavelength response to the long wavelength one in a rapid manner leading to a parallel sharp increase of $b^{\mathrm{c}}$ in Fig. 11a. The analysis of the post-bifurcation response is expected to clarify further the physics for such a "modulated" response. Even so, a precise control of $\lambda_{1}$, albeit being a very difficult task as explained previously, can lead to an interesting pattern switching with minimal energetic requirements.

\section{Results: Multi-layered structures and data-mining for minimization of critical magnetic field}

In this section, we implement an extensive data-mining exercise in an attempt to obtain combinations of structures that allow to reach a minimum critical magnetic field $b^{\mathrm{c}}$. It should be noted here that in addition to the geometrical

\footnotetext{
${ }^{8}$ One might reach regime $\mathbf{I}$ by allowing $\lambda_{1}>1$ but such loadings were not considered in the present study to keep the analysis in contact with potentially simple experimental setups.
} 
nonlinearities induced by the sinusoidal bifurcation modes, the magneto-mechanical response of the materials under study is also nonlinear.

Specifically, we consider a film comprising two layers denoted with suffixes " $f, 1$ " and " $f$, 2 " with two different topological settings; a bilayer and a sandwich film, as shown in Fig. 2 and defined in Table 1 and Table 2. With reference to this figure, we discuss first a representative bilayer film topology resting on a non-magnetic substrate. Subsequently, we perform a large scan of several parameters such as the mechanical shear moduli ratio $G^{\mathrm{f}, 2} / G^{\mathrm{f}, 1}$, $c^{\mathrm{f}, 2} / c^{\mathrm{f}, 1}, h^{\mathrm{f}, 2} / h^{\mathrm{f}, 1}$ and $c^{\mathrm{f}}$ together with $c^{\mathrm{s}}$ and $G^{\mathrm{s}}$ and all considered topologies, i.e., monolayer, bilayer and sandwich film on a substrate.

\subsection{Bilayer with one non-magnetic and slightly softer layer}

In this section, for illustration purposes, we discuss the bilayer film topology, introduced in Fig. 2 , with $c^{f, 1}=0.2$ and $c^{f, 2} / c^{f, 1}=0.0$, i.e., the second layer is non-magnetic. The overall shear moduli for the individual layers are set to $G^{\mathrm{f}, 1}=15 \mathrm{kPa}$ and $G^{\mathrm{f}, 2}=10 \mathrm{kPa}$. The underlying substrate is non-magnetic $\left(c^{\mathrm{s}}=0\right)$ and has a shear modulus $G^{\mathrm{s}}=3 \mathrm{kPa}$. We observe in Fig. 12a that the qualitative bifurcation diagrams for $b^{\mathrm{c}}$ are similar to those presented in the previous examples. Rather interestingly, increase of pre-compression along $X_{2}$ (i.e., smaller $\lambda_{2}$ ) leads to decrease of the critical magnetic field to fairly small values of $b^{\mathrm{c}}<0.15 \mathrm{~T}$ for all $\lambda_{1}$. In turn, contrary to all previously studied cases in this work, in Fig. 12b, we observe a reverse response of $\|\omega\| h^{\mathrm{f}}$. Specifically, we observe that increase of the pre-compression (decrease of $\lambda_{1}$ ), leads to decrease of the corresponding amplitude of the wave number.
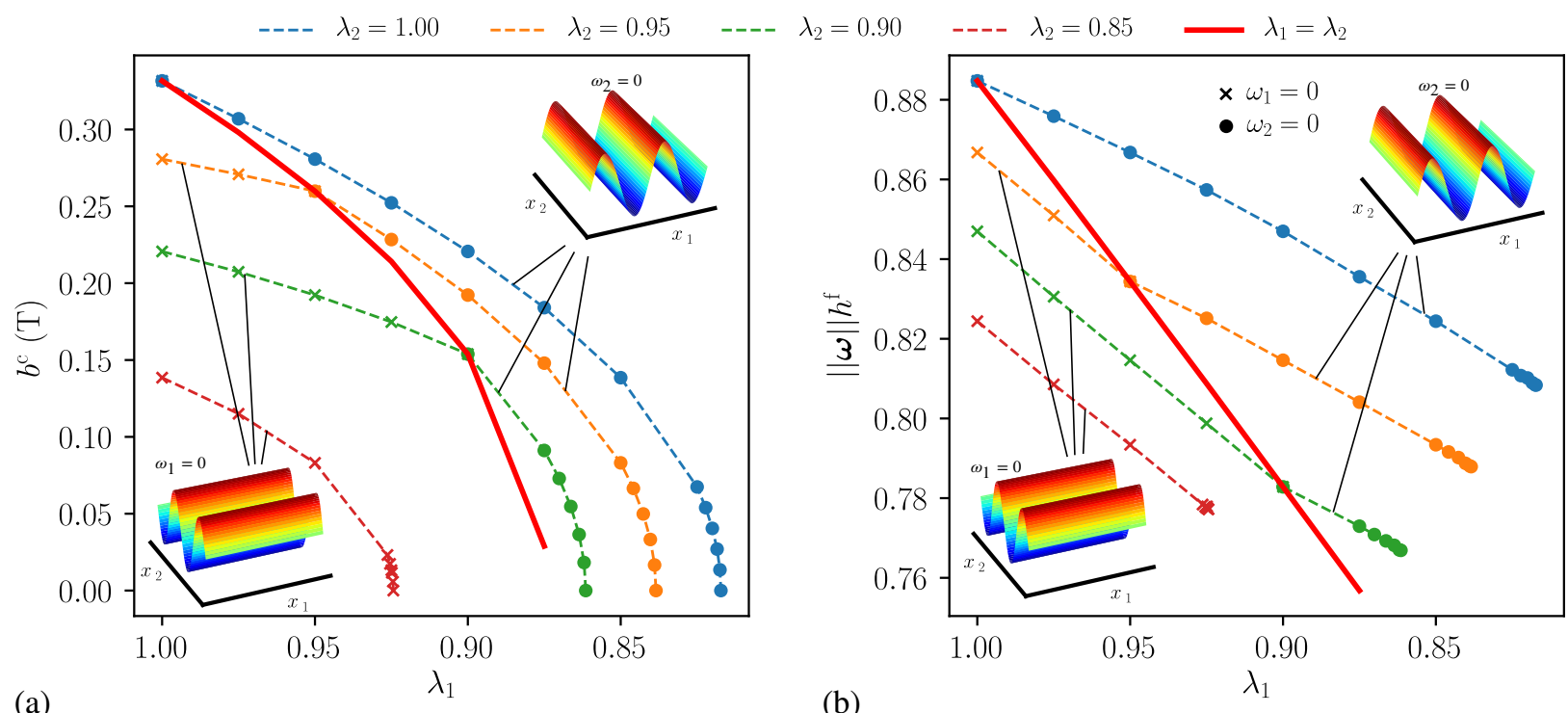

Figure 12: Effect of biaxial pre-compression for a MRE bilayer film on a passive substrate with representative parameters $c^{\mathrm{f}, 1}=0.2, c^{\mathrm{f}, 2}=0$, $h^{\mathrm{f}, 2}=h^{\mathrm{f}, 1}, G^{\mathrm{f}, 1}=15 \mathrm{kPa}, G^{\mathrm{f}, 2}=10 \mathrm{kPa}, G^{\mathrm{s}}=3 \mathrm{kPa}$ and $c^{\mathrm{s}}=0$. Bifurcation diagrams for (a) the critical magnetic field $b^{\mathrm{c}}$ and (b) the critical wave number amplitude $\|\omega\| h^{f}$ as a functions $\lambda_{1}$ for several values of $\lambda_{2}$. The solid red line marks the locus of critical states for biaxial pre-compression, across which the direction of the critical mode changes as indicated by the insets and the markers.

Furthermore, we point out that we conducted simulations of the bilayer and monolayer topologies over a wide range of parameters. Therein, we observe similar overall responses and transitions across the equi-biaxial state as for the monolayer topology underlining their fundamental nature. The agreement between the bilayer and the sandwich topologies for comparable set of parameters was found to be very close leading to responses such as the one described in Fig. 12.

\subsection{Minimizing the critical magnetic field for the case of only slight pre-compression}

This section presents a data-mining exercise with the goal of minimizing the critical magnetic field $b^{\mathrm{c}}$ by choice of the various topologies and material parameters at hand. For this purpose, an extensive scan of several geometric and model parameters is carried out. For tractability of the results and given the observations in the previous subsection, 
we focus on a single pair of stretches $\left(\lambda_{1}, \lambda_{2}\right)=(0.975,1.0)$. The slight bias in the biaxiality is enforced to avoid the special equi-biaxial case and thus allowing for easier experimental reproduction, in silico or in realitas.

Table 4: Range of parameters for the minimization of $b^{\mathrm{c}}$

\begin{tabular}{lcc}
\hline Parameter & Monolayer & Bilayer/Sandwich \\
\hline$G^{\mathrm{f}, 1}(\mathrm{kPa})$ & 10 & 10 \\
$c^{\mathrm{f}, 1}(-)$ & $0.1-0.2$ & $0.1-0.2$ \\
$G^{\mathrm{s}}(\mathrm{kPa})$ & $1-3$ & $1-3$ \\
$c^{\mathrm{s}}(-)$ & $0-0.2$ & 0 \\
$G^{\mathrm{f}, 2}(\mathrm{kPa})$ & - & $6.7-13.3$ \\
$c^{\mathrm{f}, 2}(-)$ & - & $0-0.2$ \\
$h^{\mathrm{f}, 2} / h^{\mathrm{f}, 1}(-)$ & - & $1 / 10-10$ \\
\hline
\end{tabular}

The range of parameters considered is summarized in Table 4. The magnetic parameters of the particles are kept at the same values as in the previous sections (see also Table 2).

In the following, we first investigate the effect of each topology upon the minimum critical magnetic field $b^{\mathrm{c}}$ for a given set of parameters, whereby we keep $G^{\mathrm{f}}=G^{\mathrm{f}, 1}=10 \mathrm{kPa}$ and $G^{\mathrm{s}}=3 \mathrm{kPa}$. After that, we study the effect of reducing the shear modulus of the substrate to $G^{\mathrm{s}}=1 \mathrm{kPa}$, while still keeping $G^{\mathrm{f}}=G^{\mathrm{f}, 1}=10 \mathrm{kPa}$.

Figure 13 shows collective data for a given passive substrate material with $G^{\mathbf{s}}=3 \mathrm{kPa}$.

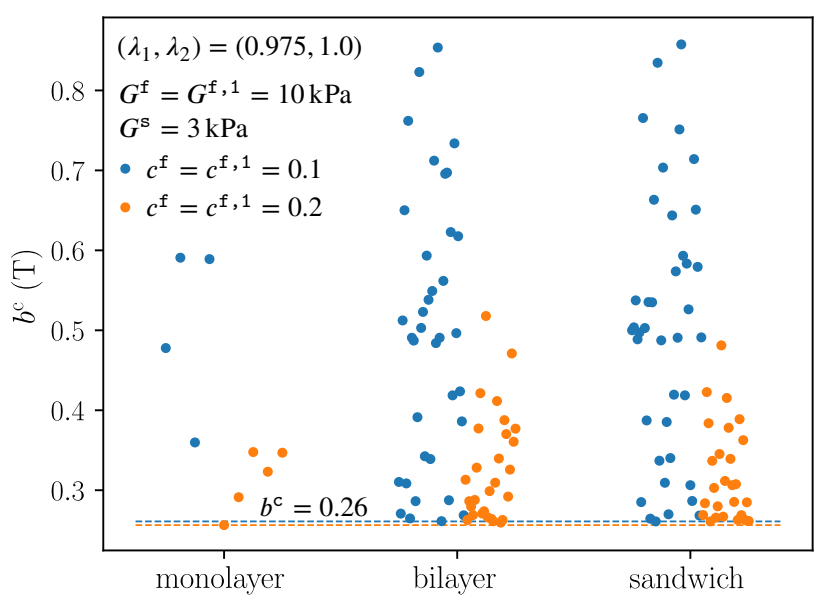

Figure 13: Minimizing the critical field $b^{c}$ for mono- and multilayer MRE films. The plot shows a wide range of results which is caused by the many possible combination of parameters.

In the case of the monolayer MRE film, we also vary the particle volume fraction in the substrate $c^{\mathrm{s}}$, whereas for the bilayer and sandwich films we set $c^{\mathrm{s}}=0$ but instead vary $c^{\mathrm{f}, 2}$, i.e. the particle volume fraction of the second film phase. Thereby, we excluded the results for equally magnetic layers, which closely resemble the monolayer case. The scatter of $b^{c}$ in Fig. 13 is extremely wide (ranging from values between $0.26 \mathrm{~T}$ to $0.85 \mathrm{~T}$ ) and demonstrates that several other parameters significantly affect the critical loads for bifurcation. In particular, we observe that certain combinations of parameters lead to a minimal critical field. Interestingly, all topologies yield practically identical minimal values near $b^{\mathrm{c}} \approx 0.26 \mathrm{~T}$. In Table 5, we report the parameter sets leading to a minimum $b^{\mathrm{c}}$ for all three topologies, e.g., monolayer, bilayer and sandwich. By close observation, we can observe that all three topologies are able to provide the smallest obtained critical magnetic field. It is, nonetheless, very difficult to extract a general rule of thumb for reaching such a low value. As we see for example in Table 5 for the sandwich topology, an interchange of particle volume fraction in the two layers forming the sandwich leads to similar responses.

Remarkably, all of these nearly optimal cases closely resemble the optimal monolayer structure, i.e., there is no substantial benefit by the use of multilayer films. In turn, we conclude that the film must be as magnetic as possible. As 
Table 5: Parameter sets for minimal critical field $b^{\mathrm{c}}$ at $\left(\lambda_{1}, \lambda_{2}\right)=(0.975,1.0)$ for $G^{\mathrm{s}}=3 \mathrm{kPa}$

\begin{tabular}{cccc} 
Monolayer with $G^{\mathrm{f}}=10 \mathrm{kPa}$ and $G^{\mathrm{s}}=3 \mathrm{k}$ \\
\hline$b^{\mathrm{c}}(\mathrm{T})$ & $\|\omega\| h^{\mathrm{f}}$ & $c^{\mathrm{s}}(-)$ & $c^{\mathrm{f}}(-)$ \\
\hline 0.256 & 0.72 & 0 & 0.2 \\
0.291 & 0.71 & 0.05 & 0.2 \\
0.323 & 0.67 & 0.1 & 0.2 \\
0.347 & 0.32 & 0.2 & 0.2 \\
0.348 & 0.58 & 0.15 & 0.2 \\
\hline
\end{tabular}

Bilayer with $G^{\mathrm{f}, 1}=10 \mathrm{kPa}, G^{\mathrm{s}}=3 \mathrm{kPa}$ and $c^{\mathrm{s}}=0$

\begin{tabular}{cccccc}
\hline$b^{\mathrm{c}}(\mathrm{T})$ & $\|\omega\| h^{\mathrm{f}}$ & $c^{\mathrm{f}, 1}(-)$ & $G^{\mathrm{f}, 2}(\mathrm{kPa})$ & $c^{\mathrm{f}, 2}(-)$ & $h^{\mathrm{f}, 2} / h^{\mathrm{f}, 1}(-)$ \\
\hline 0.259 & 0.76 & 0.2 & 6.7 & 0 & 0.1 \\
0.261 & 0.74 & 0.2 & 6.7 & 0.1 & 0.1 \\
0.261 & 0.77 & 0.1 & 6.7 & 0.2 & 10 \\
0.263 & 0.77 & 0.2 & 13.3 & 0 & 0.1 \\
0.263 & 0.80 & 0.2 & 6.7 & 0 & 0.2 \\
\hline
\end{tabular}

Sandwich with $G^{\mathrm{f}, 1}=10 \mathrm{kPa}, G^{\mathrm{s}}=3 \mathrm{kPa}$ and $c^{\mathrm{s}}=0$

\begin{tabular}{cccccc}
\hline$b^{\mathrm{c}}(\mathrm{T})$ & $\|\omega\| h^{\mathrm{f}}$ & $c^{\mathrm{f}, 1}(-)$ & $G^{\mathrm{f}, 2}(\mathrm{kPa})$ & $c^{\mathrm{f}, 2}(-)$ & $h^{\mathrm{f}, 2} / h^{\mathrm{f}, 1}(-)$ \\
\hline 0.261 & 0.77 & 0.1 & 6.7 & 0.2 & 10 \\
0.261 & 0.76 & 0.2 & 6.7 & 0 & 0.1 \\
0.262 & 0.74 & 0.2 & 13.3 & 0 & 0.1 \\
0.263 & 0.73 & 0.2 & 6.7 & 0.1 & 0.1 \\
0.263 & 0.72 & 0.2 & 13.3 & 0.1 & 0.1 \\
\hline
\end{tabular}

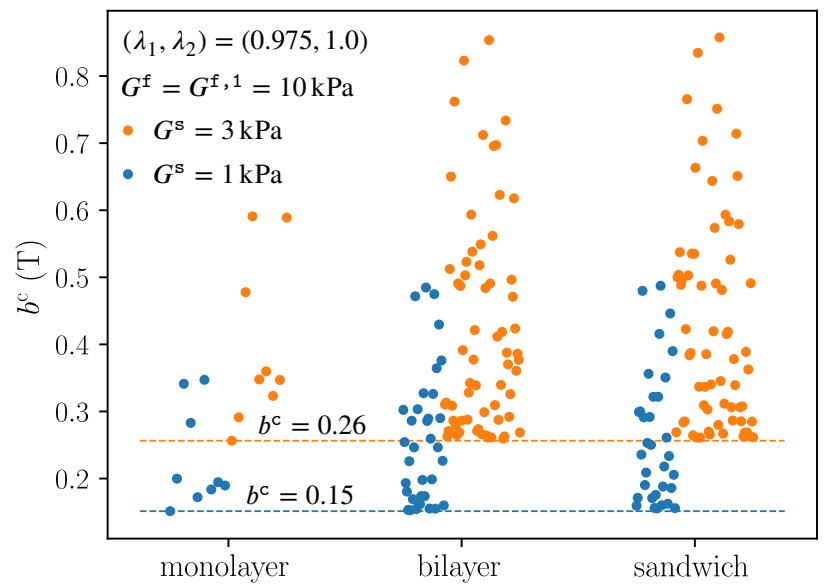

Figure 14: Effect of shear modulus of the substrate for mono- and multilayer MRE films. The drop of shear modulus translates into a drop of the minimal critical field $b^{\mathrm{c}}$ from 0.26 to $0.15 \mathrm{~T}$.

a result, the best performing multilayer topologies are those where the magnetically dominant layer almost completely covers the total volume of the film. This observation increases the relevance of the parameter studies for monolayer films in Section 4, which render fairly general guidelines for optimizing the critical field. The data for the monolayer case in Table 5 furthermore documents that structures with a magnetic substrate do not reach optimal results in terms of $b^{\mathrm{c}}$. A similar observation is also true for the bilayer and sandwich topologies and thus $c^{\mathrm{s}}=0$ in those cases. On the other hand, a magnetic substrate gives several other interesting effects such as pattern switching and for a large range of pre-compressions mechanically independent response, as discussed in the previous sections. 
Table 6: Parameter sets for minimal critical field $b^{\mathrm{c}}$ at $\left(\lambda_{1}, \lambda_{2}\right)=(0.975,1.0)$ for $G^{\mathrm{s}}=1 \mathrm{kPa}$

Monolayer with $G^{\mathrm{f}}=10 \mathrm{kPa}$ and $G^{\mathrm{s}}=1 \mathrm{kPa}$

\begin{tabular}{cccc}
\hline$b^{\mathrm{c}}(\mathrm{T})$ & $\|\omega\| h^{\mathrm{f}}$ & $c^{\mathrm{s}}(-)$ & $c^{\mathrm{f}}(-)$ \\
\hline 0.151 & 0.55 & 0 & 0.2 \\
0.172 & 0.55 & 0.05 & 0.2 \\
0.184 & 0.32 & 0.2 & 0.2 \\
0.190 & 0.52 & 0.1 & 0.2 \\
0.195 & 0.06 & 0.2 & 0.1 \\
\hline
\end{tabular}

Bilayer with $G^{\mathrm{f}, 1}=10 \mathrm{kPa}, G^{\mathrm{f}, 2}=3 \mathrm{kPa}, G^{\mathrm{s}}=1 \mathrm{kPa}$ and $c^{\mathrm{s}}=0$

\begin{tabular}{ccccc}
\hline$b^{\mathrm{c}}(\mathrm{T})$ & $\|\omega\| h^{\mathrm{f}}$ & $c^{\mathrm{f}, 1}(-)$ & $c^{\mathrm{f}, 2}(-)$ & $h^{\mathrm{f}, 2} / h^{\mathrm{f}, 1}(-)$ \\
\hline 0.153 & 0.60 & 0.2 & 0 & 0.1 \\
0.153 & 0.59 & 0.2 & 0.1 & 0.1 \\
0.155 & 0.63 & 0.2 & 0 & 0.2 \\
0.155 & 0.61 & 0.2 & 0.1 & 0.2 \\
0.155 & 0.69 & 0.1 & 0.2 & 10 \\
\hline
\end{tabular}

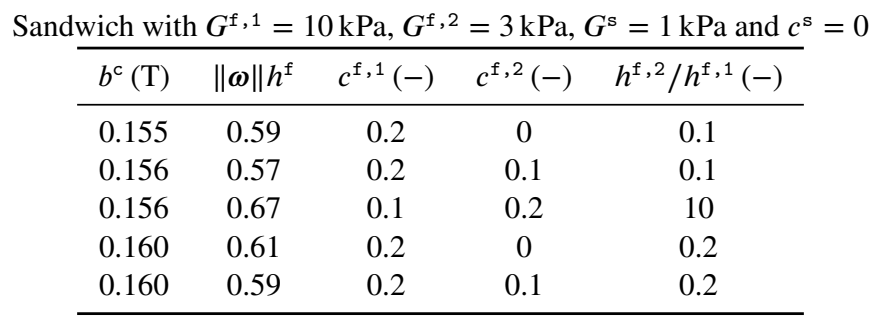

Fig. 14 discusses the effect of the substrate shear modulus $G^{\mathrm{s}}$, which is found to have a decisive effect upon $b^{\mathrm{c}}$ irrespective of other parameters ${ }^{9}$.

As expected, decrease of $G^{\mathrm{s}}$ leads to decrease of $b^{\mathrm{c}}$ from 0.26 to $0.15 \mathrm{~T}$ as well as of $\|\omega\| h^{\mathrm{f}}$ (see Table 6 and compare with Table 5). The best performing sets of parameters per film topology are summarized in Table 6.

It is mentioned here that we have deviated from the parameters in Table 4 by setting $G^{\mathrm{f}, 2}=3 \mathrm{kPa}$ in expectation of additional effects. Surprisingly, this change does not have any significant influence on computed $b^{\mathrm{c}}$ compared with that for $G^{\mathrm{f}, 2}=6.7$ to $13.3 \mathrm{kPa}$. However, what can be observed is a more pronounced difference in the wave numbers in the order of $10 \%$ between cases where $h^{\mathrm{f}, 2} / h^{\mathrm{f}, 1}<1$ and cases where $h^{\mathrm{f}, 2} / h^{\mathrm{f}, 1}>1$.

\section{Conclusion}

In this work we investigate the stability, or loss of stability, of single- and multilayer magnetorheological elastomer (MRE) films bonded to soft passive or MRE substrates. The magnetic properties of the layers and the substrate are varied with the aid of a recently proposed explicit, analytical, homogenization-guided constitutive model for MREs (Mukherjee et al., 2020), which includes explicitly the particle volume fraction as a continuum parameter. In turn, the boundary value problem considers layers that are infinite in the in-plane directions. This allows using a finite element discretization in the out-of-plane direction combined with a classical Fourier-approach in the lateral directions for the determination of the seeked critical states. It is important to mention that the finite element approach is versatile and very accurate in the present context allowing to deal with a multitude of various multi-layer-type topologies in a very convenient manner. For instance, by simply changing the discretization and the material properties in each layer, the analysis is readily carried out since mechanical and magnetic continuity is treated in a straightforward manner by

\footnotetext{
${ }^{9}$ This effect is related to those discussed in Fig. 6 and Fig. 7, where the latter also discussed the ratio $G^{\mathrm{s}} / G^{\mathrm{f}}$ with $G^{\mathrm{s}}$.
} 
the finite element formalism. This allowed us to carry out a very large number of calculations and examine various multilayer topologies with varying material properties. As a result, a data-mining exercise has led to optimal critical magnetic fields for all topologies considered.

In the first set of examples, we discuss a representative MRE layer on a soft passive (i.e. non-magnetic) substrate under general biaxial pre-compression states. Specifically, we find that biaxial pre-compression leads to a significant reduction of the critical magnetic field when compared to that for uni-axial pre-compression. Moreover, we observe a sharp transition in the bifurcation response - both in the critical magnetic field and corresponding amplitude of the wave numbers - by crossing the purely equi-biaxial stretch state. This transition leads also to a change of the direction of the wave vector. In the sequence, we explore the effect of the various material parameters such as the mechanical stiffness of the film and substrate as well as the volume fraction of the magnetic particles in the film leading to different magnetic properties. Specifically, we show that the mechanical stiffness of the film alone (as this is described by the shear modulus due to the considered quasi-incompressibility) has significant effects on the bifurcation response. Specifically, as the film becomes mechanically stiffer, the range of pre-compressions that enable an unstable response shrinks to a very narrow band lying very close to the mechanical bifurcation. This effect has been missed in the study of Danas and Triantafyllidis (2014) and simply indicates that softer films such as the one used in Psarra et al. (2019) have a very wide range of unstable response which can be efficiently modulated by the applied pre-compressions.

Another key result is the characterization of the effect of the volume fraction of magnetic particles contained in the MRE film, since they affect strongly the magnetic properties of the film as well as the mechanical ones. Specifically, we find that increase of the particle volume fraction in the film leads to a monotonic decrease of the critical magnetic field for all pre-compressions applied in this work (at least up to volume fractions examined, i.e., 40vol\% but only $25 \mathrm{vol} \%$ shown in the present study). This implies that the parallel increase in stiffness of the film with the addition of more particles, which would otherwise lead to increase of the critical magnetic field, is not sufficient to overcome the significant increase of magnetic susceptibility. In addition, this decrease of the critical magnetic field is accompanied by a decrease of the corresponding wave number.

The second set of results investigates for the first time the influence of a magnetic substrate at the same time with a magnetic film. This is achieved by varying the magnetic particle volume fraction in those materials allowing for a relative effect of the corresponding mechanical and magnetic responses. We find for instance, that a maximum value of the critical magnetic field is obtained when the particle volume fraction in the film and the substrate is fairly equal, while the film is still three to four times stiffer mechanically than the substrate. More interestingly, we observe a very strong effect of the substrate particle volume fraction on the critical magnetic fields and wave numbers that was not observed for any other parameter before. For a magnetically dominant substrate, one could clearly distinguish a mechanically and magnetically governed regime both in the critical magnetic field and the corresponding wave numbers. Within the mechanically governed regime, the critical magnetic field is very sensitive to the level of precompression, whereas in the magnetically governed regime, the critical field is, in comparison, almost independent of the pre-compression. The transition between the two regimes is accompanied with very sharp changes (almost a jump) in the wave numbers within a small range of pre-compression. The quantitative changes in the wave numbers are extremely pronounced spanning very long wavelength to very short wavelength response of the surface pattern. This last effect could be a possible candidate mechanism for magneto-mechanical surface-pattern switching with minimal energy input. Nonetheless, a very precise design of the magneto-mechanical device is required to control accurately the imposed magnetic loads and pre-compression.

The third and final set of results is concerned with the optimization of the critical magnetic field for given prestretches and for three different topologies, e.g., a monolayer, a bilayer and a sandwich film bonded to a substrate (see Fig. 2 for a graphical representation). The optimization exercise is achieved in terms of a data-mining exercise allowing to deal with both the geometrical nonlinearities due to the sinusoidal eigenmodes as well as the nonlinear magneto-mechanical response of the constituents. For the cases investigated, we find as a main result that the minimum critical field is obtained for single-layer and single-layer-type topologies. In connection with this, a large number of key parameters is varied allowing to show that a very large range of critical magnetic fields can be reached by arbitrarily changing the particle volume fraction in the film, the mechanical stiffness of the constituents, as well as the various topologies of the layers inside the film. Also, it is shown that an intuitive approach to the problem cannot lead to a well-designed experimental device for surface patterning.

From a more general perspective, many of the multilayer structures discussed in the present study might exhibit interesting features at some critical state and in particular in the post bifurcation regime. Nonetheless, the very large num- 
ber of geometric and material parameters makes a full post-bifurcation numerical or experimental study prohibitive. In turn, the present bifurcation results allow to select interesting and non-intuitive cases for further investigations and prepare the ground for experimental and numerical studies exploring the post-bifurcation regime.

\section{Acknowledgements}

The authors would like to acknowledge support from the European Research Council (ERC) under the European Union's Horizon 2020 research and innovation program (grant agreement No 636903 - MAGNETO). The computational part of this work was also supported by the ANR, France under contract number ANR-10-EQPX-37.

\section{Appendix A. Effect of film thickness for single- and multilayer topologies}

We know from the literature in the purely mechanical response of a film/substrate system, that, given a substrate that is much thicker than the film, the actual film thickness does not affect the bifurcation behavior. This is the reason why we have mainly focused on thin films in the main part of the study. For completeness, however, we provide in this appendix a small study where we increase the film thickness up to values similar to the thickness of the substrate as shown in Fig. A.15a. We study a similar effect in the context films with bilayer and sandwich structure, as shown in Figs A.15b and A.15c, respectively.

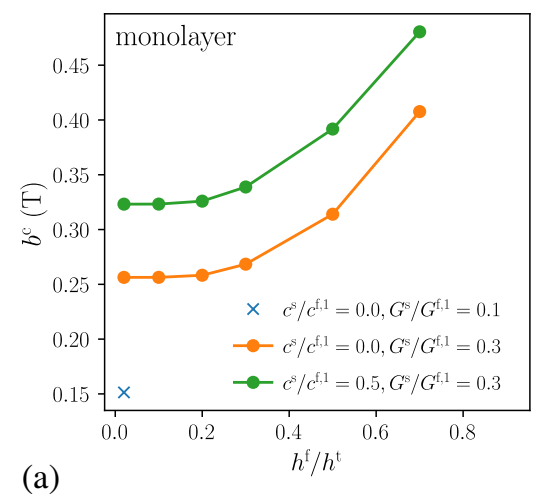

(a)

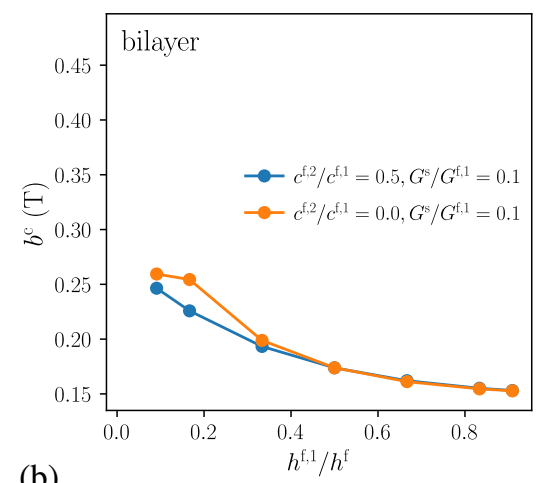

(b)

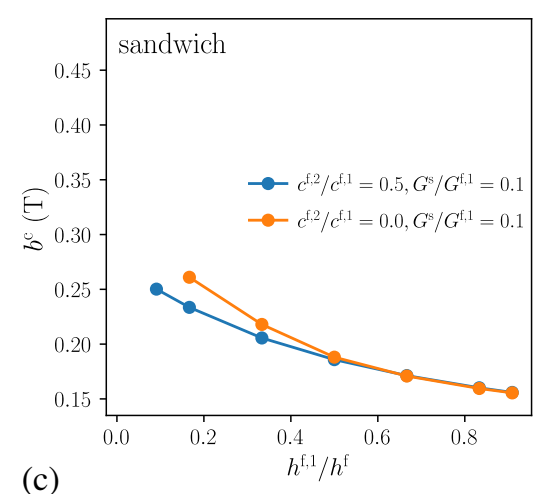

Figure A.15: Effect of the relative film thickness on the bifurcation behavior for mono- and multilayer MRE films bonded onto a passive substrate. The reference thickness for the monolayer is the total height of the film-substrate structure, that is $h^{\mathrm{f}}$. For the multiphase films we employ thickness of film itself as reference. The parameters for the monolayer film are $G^{\mathrm{f}}=10 \mathrm{kPa}, c^{\mathrm{f}}=0.2$. For the bilayer and the sandwich films we have $G^{\mathrm{f}, 1}=10 \mathrm{kPa}, G^{\mathrm{f}, 2}=3 \mathrm{kPa}$ and $G^{\mathrm{s}}=1 \mathrm{kPa}$.

We emphasize that in Fig. A.15 the reference thickness for the monolayer is the total height of the film-substrate structure, that is $h^{\mathrm{f}}$, whereas for multilayer films (see Fig. 2) the total film thickness serves as a reference. The choices of the shear moduli for the monolayer are $G^{\mathrm{f}}=10 \mathrm{kPa}$ and $G^{\mathrm{s}}=3 \mathrm{kPa}$, while we for the multilayer cases employ $G^{\mathrm{f}, 1}=10 \mathrm{kPa}, G^{\mathrm{f}, 2}=3 \mathrm{kPa}$ and $G^{\mathrm{s}}=1 \mathrm{kPa}$. By virtue of these values, a very thin film phase " 1 " $\left(h^{\mathrm{f}, 2} / h^{\mathrm{f}, 1} \gg\right.$ $\left.1 \Leftrightarrow h^{\mathrm{f}, 1} / h^{\mathrm{f}} \ll 1\right)$ corresponds to the monolayer setting because in that case the substrate does not play a role any more. Conversely, if the thickness of the monolayer increases, the rigid vertical support of the substrate acts against the vertical displacement of the magnetic layer and thus increases the critical magnetic field. Another interesting observation in Fig. A.15 is that a slightly magnetic substrate increases the critical magnetic field. Furthermore, a magnetic second film phase decreases $b^{\mathrm{c}}$ when $h^{\mathrm{f}, 2} / h^{\mathrm{f}, 1}$ is significant. This can be explained by the corresponding increase of the effective magnetic properties of the multilayer films. This is in agreement with Fig. 13, where we show different sets of parameters yielding a critical field $b^{c}$ close to the best minimum obtained.

\section{Appendix B. Scanning for critical states}

In this section, we detail the procedures employed for finding the critical bifurcation states. At the very heart of the procedure is the computation of the minimal eigenvalue of $\mathbf{K}$ (see (34)). For this operation, we use the iterative eigenvalue solver package ARPACK (Lehoucq et al., 1998) provided by scipy (Virtanen et al., 2020). Since we 
aim for eigenvalues close to zero, we let ARPACK compute the ten smallest eigenvalues ${ }^{10}$ using a shift-invert spectral transform in "normal" mode and shift parameter $\sigma=0$. From these, we then select the smallest eigenvalue denoted $\Lambda^{\min }\left(\boldsymbol{\Omega}, b^{\infty}, \lambda_{1}, \lambda_{2}\right)$ and by that (approximately) solve the inner minimization problem in (34).

The boundary conditions of the discretized fields in the eigenvalue problem are essentially the same as for the continuous problem (15) with some minor differences. Specifically, due to the finite extent of the domain in the $X_{3}$, we have

$$
\Delta \boldsymbol{\varphi}_{h}(\mathbf{X})=\mathbf{0} \quad X_{3} \text { for } X_{3} \notin(\mathcal{B} \cup \partial \mathcal{B})
$$

and

$$
\Delta \mathbf{A}_{h}(\mathbf{X})=0 \text { for } \quad X_{3}=X_{3}^{\min } \vee X_{3}=X_{3}^{\max }
$$

which can be applied on the actual finite element degrees of freedom in a direct manner. Note that due to the orthogonality of the trigonometric functions, these conditions have to be fulfilled per mode. Thus, they are applied in the eigenvalue problem of (34) for each $\boldsymbol{\Omega}$. In addition, we remove the coefficients of the zero-modes similar to the corresponding problems with reduced dimensionality, i.e.,

$$
\Delta \check{\varphi}_{i}=0 \quad \text { if } \quad \omega_{i}=0
$$

and

$$
\Delta \check{A}_{j}=\Delta \check{A}_{k}=0 \quad \text { if } \quad \omega_{i}=0 \quad(i \neq j \neq k) .
$$

Moreover, since $\sin (0)=0$, we set the sin-coefficients $\Delta \widehat{\mathbf{A}}^{\mathrm{s}}(\mathbf{0})=\Delta \hat{\boldsymbol{\varphi}}^{\mathrm{s}}(\mathbf{0})=0$. For $\omega_{1} \rightarrow 0$ or $\omega_{2} \rightarrow 0$, we face the problem that the system becomes numerically under-constrained. This particular case corresponds to very large wavelengths (quasi-rigid modes) and are excluded from the domain of admissible $\boldsymbol{\Omega}$ since they lead to numerically singular systems for sufficiently small $\|\mathbf{\Omega}\|$. Since arbitrarily long (but not infinite) wavelength modes are not of practical interest in the present study their exclusion does affect the present results. An example of such a case is the vanishing mechanical stiffness of the substrate phase. In Danas and Triantafyllidis (2014), this case is shown to lead to a zero critical magnetic field and zero wave number. Such limiting theoretical cases are not studied in the present study. Instead, minor modifications in the present problem can readily allow their investigation.

The next step is to minimize the smallest eigenvalues $\Lambda^{\min }\left(\boldsymbol{\Omega}, b^{\infty}, \lambda_{1}, \lambda_{2}\right)$ over the entire domain of $\boldsymbol{\Omega}$. This is a delicate problem since we expect several local minima and thus have to do global optimization in two dimensions in general. However, for the purely mechanical case and not too complicated materials ${ }^{11}$ general $\left(\Omega_{1}, \Omega_{2}\right)$ are only expected under equi-biaxial loading, i.e. when $\lambda_{1}=\lambda_{2}$. Moreover, in this case there is a set of solutions for $\mathbf{\Omega}$ which forms a circle $\|\mathbf{\Omega}\|=$ const (Chen and Hutchinson, 2004). On the other hand, if $\lambda_{1}<\lambda_{2} \leq 1$, then $\boldsymbol{\Omega}=\left(\Omega_{1}, 0\right)$ and vice-versa. In such a setting, the search for $\boldsymbol{\Omega}$ is only in one dimension. However, it is not cautious to extend this by assumption to the case of MREs under magneto-mechanical loading. Therefore, we employ the "Simplicial Homology Global Optimization" (SHGO) algorithm (Endres et al., 2018) included in scipy for two-dimensional but also one-dimensional searches for $\boldsymbol{\Omega}$. In both cases we opt for a sampling via Sobol sequences Sobol (1967) with 20 points by default for minimization in two dimensions $(\boldsymbol{\Omega})$ and 40 points for minimization in one dimension $\left(\Omega_{1}\right.$ or $\left.\Omega_{2}\right)$.

We remark that finding the global optimum with the SHGO basically depends on the sampling of the function that is minimized. In a number of tests we found that 20 or 40, respectively, Sobol points offer a good compromise between computational cost and probability in finding the global optimum. Another possibility is to employ the simplicial sampling of SHGO combined with a sufficient number of refinements of the initial sampling. For our test cases, four sampling iterations lead to sufficient performance. However, a fifth iteration significantly increased the computational effort without changing results. Interestingly, the global optimization in two dimensions often lead to a better performance even if the actual solution is the same as for the one-dimensional search. One possible reason for this is that two local minima in one dimension could actually be connected in the plane such that less global sampling points are needed right from the beginning. As an internal (local) solver we used "COBYLA" to which we passed on the

\footnotetext{
${ }^{10}$ Computing the ten eigenvalues closest to zero turns out to be a good compromise between the computational effort and the probability of missing any negative eigenvalue. Given that we usually start from a stable state and iterate towards loss of stability, this issue is not of major concern.

${ }^{11}$ Under special conditions this does not hold (Carfagna et al., 2017).
} 
bounds given to SHGO. Tolerances were set to $1 \times 10^{-8}$. The results of this "second" minimization shall be denoted $\boldsymbol{\Omega}^{\min }\left(b^{\infty}, \lambda_{1}, \lambda_{2}\right)$ and $\bar{\Lambda}^{\min }\left(b^{\infty}, \lambda_{1}, \lambda_{2}\right)=\Lambda^{\min }\left(\boldsymbol{\Omega}^{\min }\left(b^{\infty}, \lambda_{1}, \lambda_{2}\right), b^{\infty}, \lambda_{1}, \lambda_{2}\right)$.

Being in possession of a procedure yielding $\left\{\boldsymbol{\Omega}^{\min }\left(b^{\infty}, \lambda_{1}, \lambda_{2}\right), \bar{\Lambda}^{\min }\left(b^{\infty}, \lambda_{1}, \lambda_{2}\right)\right\}$, we either choose $\left\{\lambda_{1}, \lambda_{2}\right\}$ and search for $b^{\infty}$ or choose $\left\{b^{\infty}, \lambda_{i}\right\}$ and search for $\lambda_{j}$ such that $\bar{\Lambda}^{\text {min }}=0$ by scipy's "brentq" root-finding algorithm, which is a modified version of the classical method (Brent, 2013). In both cases the result is a critical state $\left(b^{\mathrm{c}}, \lambda_{1}^{\mathrm{c}}, \lambda_{2}^{\mathrm{c}}\right)$. Also for this algorithm, we set all tolerances to $1 \times 10^{-8}$, whereby our tests cases have not been sensitive to the precise values of these tolerances. Algorithm 1 summarizes the high-level scanning and solution strategy.

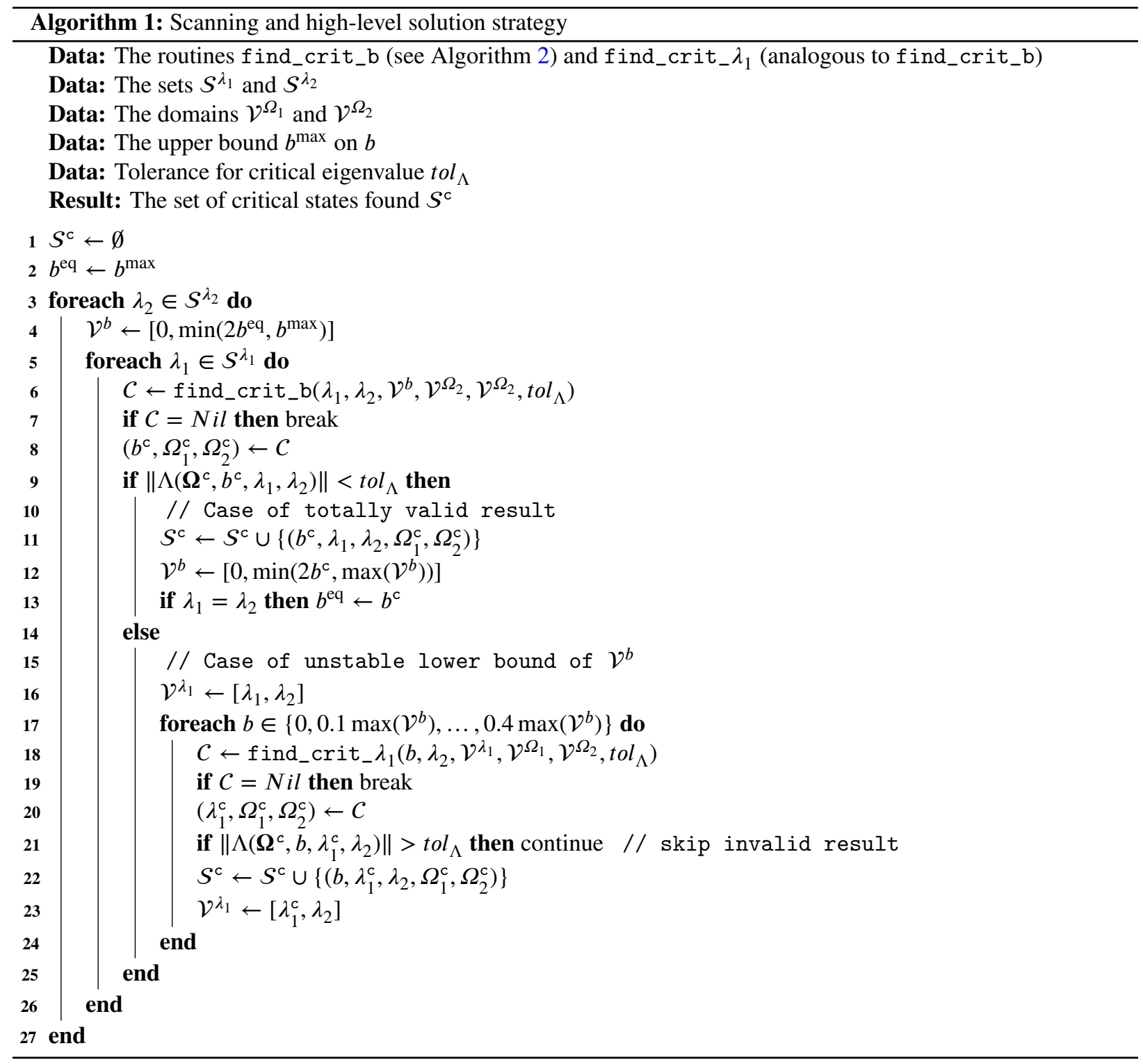

We close this section with some remarks concerning the scanning procedure:

- For the two-dimensional search with SHGO we work in the logarithmic frequency space. This leads to better sampling of the search domain. Otherwise, the range of reasonably small frequencies is tendentiously undersampled. By contrast, we did not observe significant differences between standard and logarithmic search in one dimension. This is probably caused by larger number of sampling points required in this case. 


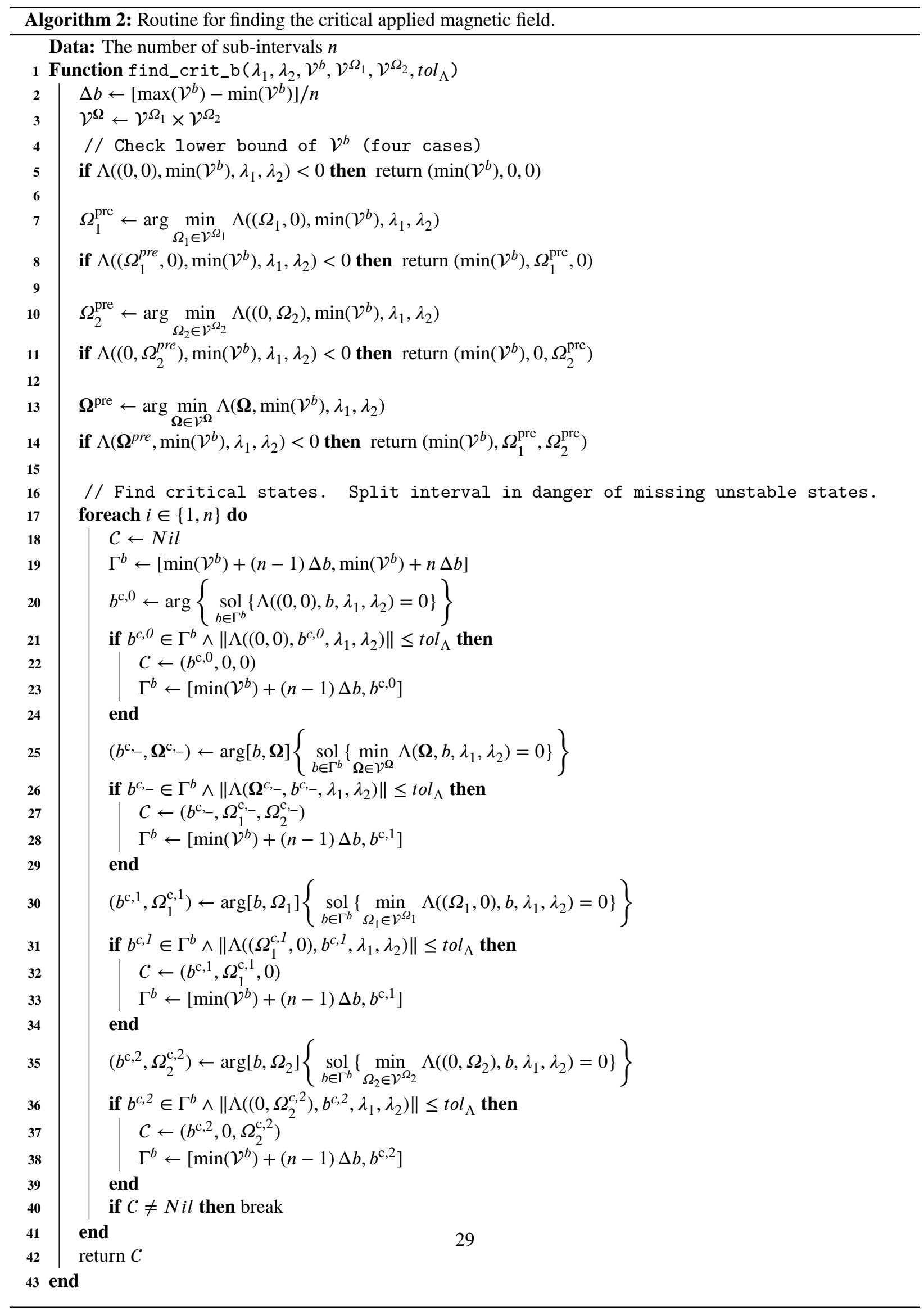


- In case of unexpected results, e.g., a detected change in sign of $\Lambda^{\min }$ but $\bar{\Lambda}^{\min }>t o l_{\Lambda}$, we repeat the procedure with an increased number of global sampling points in SHGO. This is a quite heuristic but nonetheless simple and efficient implementation detail and thus is not reflected in any line of the Algorithm 1 or Algorithm 2.

- As another implementation detail, we perform additional checks for possibly missed instabilities. For this purpose, we compute $\bar{\Lambda}^{\text {min }}$ for four equidistant "check points" within the search interval for $b^{\mathrm{c}}$ or $\lambda_{1}^{\mathrm{c}}$, respectively. In case of a negative $\bar{\Lambda}^{\mathrm{min}}$ the search for the critical load is repeated with new bounds derived from these "check points". Again, we did not analyze further the necessity of these checks in our production runs.

- Both Algorithm 1 and Algorithm 2 are independent of our choice for SHGO and brentq.

- It is worth mentioning that we have also tried to employ SHGO for directly minimizing $\|\Lambda\|$ over $(b, \mathbf{\Omega})$ instead of (inner) minimization combined with (outer) root finding. However, this did not work for us.

Algorithm parameters. We employ by default:

$$
\begin{aligned}
\mathcal{S}^{\lambda_{1}} & =\mathcal{S}^{\lambda_{2}}=\{1.0,0.975, \ldots, 0.7\}, \\
\mathcal{V}^{\Omega_{1}} & =\mathcal{V}^{\Omega_{1}}=\left\{\frac{2 \pi}{200 h^{\mathrm{f}}}, \frac{2 \pi}{1 h^{\mathrm{f}}}\right\} \mathrm{mm}^{-1}, \\
b^{\max } & =1.0 \text { and } \\
\text { tol }_{\Lambda} & =1 \times 10^{-9},
\end{aligned}
$$

where $h^{\mathrm{t}}$ and $h^{\mathrm{f}}$ are described in Table 1 .

\section{References}

Allen, H.G., 1969. Analysis and Design of Structural Sandwich Panels: The Commonwealth and International Library: Structures and Solid Body Mechanics Division. Pergamon Press.

Audoly, B., Boudaoud, A., 2008. Buckling of a stiff film bound to a compliant substrate-Part I:. Journal of the Mechanics and Physics of Solids 56, 2401-2421. doi:10.1016/j.jmps.2008.03.003

Biot, M.A., 1963. Surface instability of rubber in compression. Applied Scientific Research, Section A 12, 168-182. doi:10.1007/BF03184638.

Bodelot, L., Voropaieff, J.P., Pössinger, T., 2018. Experimental investigation of the coupled magneto-mechanical response in magnetorheological elastomers. Experimental Mechanics 58, 207-221. doi:10.1007/s11340-017-0334-7.

Brent, R.P., 2013. Algorithms for Minimization Without Derivatives. Courier Corporation.

Brown, W.F.J., 1966. Magnetoelastic Interactions. Springer Tracts in Natural Philosophy, Springer-Verlag, Berlin Heidelberg.

Bustamante, R., Dorfmann, A., Ogden, R., 2008. On Variational Formulations in Nonlinear Magnetoelastostatics. Mathematics and Mechanics of Solids 13, 725-745. doi:10.1177/1081286507079832.

Cai, S., Breid, D., Crosby, A.J., Suo, Z., Hutchinson, J.W., 2011. Periodic patterns and energy states of buckled films on compliant substrates. Journal of the Mechanics and Physics of Solids 59, 1094-1114. doi:10.1016/j.jmps.2011.02.001.

Cai, Z., Fu, Y., 1999. On the imperfection sensitivity of a coated elastic half-space. Proceedings of the Royal Society of London. Series A: Mathematical, Physical and Engineering Sciences 455, 3285-3309. URL: http://dx.doi.org/10.1098/rspa.1999.0451, doi:10.1098/ rspa.1999.0451.

Cao, Y., Hutchinson, J.W., 2012a. From wrinkles to creases in elastomers: the instability and imperfection-sensitivity of wrinkling. Proceedings of the Royal Society A: Mathematical, Physical and Engineering Sciences 468, 94-115. doi:10.1098/rspa.2011.0384.

Cao, Y., Hutchinson, J.W., 2012b. Wrinkling Phenomena in Neo-Hookean Film/Substrate Bilayers. Journal of Applied Mechanics 79 . doi:10. 1115/1.4005960.

Carfagna, M., Destrade, M., Gower, A.L., Grillo, A., 2017. Oblique wrinkles. Philosophical Transactions of the Royal Society A: Mathematical, Physical and Engineering Sciences 375, 20160158. doi:10.1098/rsta.2016.0158.

Chakrabarti, A., Mora, S., Richard, F., Phou, T., Fromental, J.M., Pomeau, Y., Audoly, B., 2018. Selection of hexagonal buckling patterns by the elastic Rayleigh-Taylor instability. Journal of the Mechanics and Physics of Solids 121, 234-257. doi:10.1016/j.jmps . 2018.07.024.

Chen, X., Hutchinson, J.W., 2004. Herringbone Buckling Patterns of Compressed Thin Films on Compliant Substrates. Journal of Applied Mechanics 71, 597-603. doi:10.1115/1.1756141.

Danas, K., 2017. Effective response of classical, auxetic and chiral magnetoelastic materials by use of a new variational principle. Journal of the Mechanics and Physics of Solids 105, 25-53. doi:10.1016/j.jmps.2017.04.016.

Danas, K., Kankanala, S.V., Triantafyllidis, N., 2012. Experiments and modeling of iron-particle-filled magnetorheological elastomers. Journal of the Mechanics and Physics of Solids 60, 120-138. doi:10.1016/j.jmps . 2011.09.006.

Danas, K., Mukherjee, D., Haldar, K., Triantafyllidis, N., 2019. Bifurcation analysis of twisted liquid crystal bilayers. Journal of the Mechanics and Physics of Solids 123, 61-79. doi:10.1016/j.jmps.2018.09.008.

Danas, K., Triantafyllidis, N., 2014. Instability of a magnetoelastic layer resting on a non-magnetic substrate. Journal of the Mechanics and Physics of Solids 69, 67-83. doi:10.1016/j.jmps.2014.04.003. 
Destrade, M., Ní Annaidh, A., Coman, C.D., 2009. Bending instabilities of soft biological tissues. International Journal of Solids and Structures 46, 4322-4330. doi:10.1016/j.ijsolstr.2009.08.017.

Dorfmann, A., Ogden, R.W., 2003. Magnetoelastic modelling of elastomers. European Journal of Mechanics-A/Solids 22, 497-507. doi:10 . 1007/ s00707-003-0061-2.

Endres, S.C., Sandrock, C., Focke, W.W., 2018. A simplicial homology algorithm for Lipschitz optimisation. Journal of Global Optimization 72, 181-217. doi:10.1007/s10898-018-0645-y.

Fu, Y.B., Ciarletta, P., 2015. Buckling of a coated elastic half-space when the coating and substrate have similar material properties. Proceedings of the Royal Society A: Mathematical, Physical and Engineering Sciences 471, 20140979. URL: http://dx.doi.org/10.1098/rspa.2014. 0979, doi:10.1098/rspa.2014.0979.

Huang, Z.Y., Hong, W., Suo, Z., 2005. Nonlinear analyses of wrinkles in a film bonded to a compliant substrate. Journal of the Mechanics and Physics of Solids 53, 2101-2118. doi:10.1016/j . jmps . 2005.03.007.

Hutchinson, J.W., 2013. The role of nonlinear substrate elasticity in the wrinkling of thin films. Philosophical Transactions of the Royal Society A: Mathematical, Physical and Engineering Sciences 371, 20120422. doi:10.1098/rsta.2012.0422.

Javili, A., Chatzigeorgiou, G., Steinmann, P., 2013. Computational homogenization in magneto-mechanics. International Journal of Solids and Structures 50, 4197-4216. doi:10.1016/j.ijsolstr.2013.08.024.

Kalina, K.A., Brummund, J., Metsch, P., Kästner, M., Borin, D.Y., Linke, J.M., Odenbach, S., 2017. Modeling of magnetic hystereses in soft MREs filled with NdFeB particles. Smart Materials and Structures 26, 105019. doi:10.1088/1361-665X/aa7f81.

Kankanala, S.V., 2007. On finitely strained magnetoelastic solids. Ph.D. thesis. University of Michigan.

Kankanala, S.V., Triantafyllidis, N., 2004. On finitely strained magnetorheological elastomers. Journal of the Mechanics and Physics of Solids 52, 2869-2908. doi:10.1016/j.jmps.2004.04.007.

Keip, M.A., Rambausek, M., 2016. A multiscale approach to the computational characterization of magnetorheological elastomers. International Journal for Numerical Methods in Engineering 107, 338-360. doi:10.1002/nme.5178.

Keip, M.A., Rambausek, M., 2017. Computational and analytical investigations of shape effects in the experimental characterization of magnetorheological elastomers. International Journal of Solids and Structures 121, 1-20. doi:10.1016/j.ijsolstr.2017.04.012.

Keip, M.A., Sridhar, A., 2019. A variationally consistent phase-field approach for micro-magnetic domain evolution at finite deformations. Journal of the Mechanics and Physics of Solids 125, 805-824. doi:10.1016/j.jmps.2018.11.012.

Kim, Y., Parada, G.A., Liu, S., Zhao, X., 2019. Ferromagnetic soft continuum robots. Science Robotics 4. doi:10.1126/scirobotics . aax7329.

Kim, Y., Yuk, H., Zhao, R., Chester, S.A., Zhao, X., 2018. Printing ferromagnetic domains for untethered fast-transforming soft materials. Nature 558, 274-279. doi:10.1038/s41586-018-0185-0.

Lefèvre, V., Danas, K., Lopez-Pamies, O., 2017. A general result for the magnetoelastic response of isotropic suspensions of iron and ferrofluid particles in rubber, with applications to spherical and cylindrical specimens. Journal of the Mechanics and Physics of Solids $107,343-364$. doi: $10.1016 / j \cdot j m p s .2017 .06 .017$.

Lefèvre, V., Danas, K., Lopez-Pamies, O., 2020. Two families of explicit models constructed from a homogenization solution for the magnetoelastic response of MREs containing iron and ferrofluid particles. International Journal of Non-Linear Mechanics 119, 103362. doi:10.1016/ $j$. i jnonlinmec.2019.103362.

Lehoucq, R.B., Sorensen, D.C., Yang, C., 1998. ARPACK Users' Guide: Solution of Large-scale Eigenvalue Problems with Implicitly Restarted Arnoldi Methods. SIAM.

Liu, F., Xu, F., Fu, C., 2019. Orientable wrinkles in stretched orthotropic films. Extreme Mechanics Letters 33, 100579. doi:10.1016/j .eml. 2019.100579.

Lopez-Pamies, O., Goudarzi, T., Danas, K., 2013. The nonlinear elastic response of suspensions of rigid inclusions in rubber: II-A simple explicit approximation for finite-concentration suspensions. Journal of the Mechanics and Physics of Solids 61, 19-37. doi:10.1016/j.jmps.2012. 08.013.

Moon, F.C., Pao, Y.H., 1969. Vibration and Dynamic Instability of a Beam-Plate in a Transverse Magnetic Field. Journal of Applied Mechanics 36, 92-100. doi:10.1115/1.3564592.

Mukherjee, D., Bodelot, L., Danas, K., 2020. Microstructurally-guided explicit continuum models for isotropic magnetorheological elastomers with iron particles. International Journal of Non-Linear Mechanics 120, 103380. doi:10.1016/j.ijnonlinmec.2019.103380.

Otténio, M., Destrade, M., Ogden, R.W., 2008. Incremental Magnetoelastic Deformations, with Application to Surface Instability. Journal of Elasticity 90, 19-42. doi:10.1007/s10659-007-9120-6.

Pocivavsek, L., Dellsy, R., Kern, A., Johnson, S., Lin, B., Lee, K.Y.C., Cerda, E., 2008. Stress and Fold Localization in Thin Elastic Membranes. Science 320, 912-916. doi:10.1126/science.1154069.

Psarra, E., Bodelot, L., Danas, K., 2017. Two-field surface pattern control via marginally stable magnetorheological elastomers. Soft Matter 13, 6576-6584. doi:10.1039/C7SM00996H.

Psarra, E., Bodelot, L., Danas, K., 2019. Wrinkling to crinkling transitions and curvature localization in a magnetoelastic film bonded to a nonmagnetic substrate. Journal of the Mechanics and Physics of Solids 133, 103734. doi:10.1016/j.jmps.2019.103734.

Sigaeva, T., Mangan, R., Vergori, L., Destrade, M., Sudak, L., 2018. Wrinkles and creases in the bending, unbending and eversion of soft sectors. Proceedings of the Royal Society A: Mathematical, Physical and Engineering Science 474, 20170827. doi:10.1098/rspa.2017.0827.

Sobol, I.M., 1967. On the distribution of points in a cube and the approximate evaluation of integrals. Zhurnal Vychislitel'noi Matematiki i Matematicheskoi Fiziki 7, 784-802.

Su, Y., Wu, B., Chen, W., Destrade, M., 2019. Finite bending and pattern evolution of the associated instability for a dielectric elastomer slab. International Journal of Solids and Structures 158, 191-209. URL: http://www.sciencedirect.com/science/article/pii/ S0020768318303640, doi:10.1016/j.ijsolstr.2018.09.008.

Su, Y., Wu, B., Chen, W., Destrade, M., 2020. Pattern evolution in bending dielectric-elastomeric bilayers. Journal of the Mechanics and Physics of Solids 136, 103670. URL: http://www.sciencedirect.com/science/article/pii/S0022509619301978, doi:10.1016/j .jmps . 2019.07.013.

Sun, J.Y., Xia, S., Moon, M.W., Oh, K.H., Kim, K.S., 2012. Folding wrinkles of a thin stiff layer on a soft substrate. Proceedings of the Royal 
Society A: Mathematical, Physical and Engineering Sciences 468, 932-953. doi:10.1098/rspa. 2011.0567.

Triantafyllidis, N., Needleman, A., 1980. An Analysis of Wrinkling in the Swift Cup Test. Journal of Engineering Materials and Technology 102, 241-248. doi:10.1115/1.3224806.

Virtanen, P., Gommers, R., Oliphant, T.E., Haberland, M., Reddy, T., Cournapeau, D., Burovski, E., Peterson, P., Weckesser, W., Bright, J., van der Walt, S.J., Brett, M., Wilson, J., Jarrod Millman, K., Mayorov, N., Nelson, A.R.J., Jones, E., Kern, R., Larson, E., Carey, C., Polat, İ., Feng, Y., Moore, E.W., Vand erPlas, J., Laxalde, D., Perktold, J., Cimrman, R., Henriksen, I., Quintero, E.A., Harris, C.R., Archibald, A.M., Ribeiro, A.H., Pedregosa, F., van Mulbregt, P., Contributors, S..., 2020. SciPy 1.0: Fundamental Algorithms for Scientific Computing in Python. Nature Methods 17,261-272. doi:https://doi.org/10.1038/s41592-019-0686-2.

Wang, Q., Zhao, X., 2014. Phase Diagrams of Instabilities in Compressed Film-Substrate Systems. Journal of Applied Mechanics 81, 051004105100410. doi:10.1115/1.4025828.

Xu, F., Potier-Ferry, M., Belouettar, S., Cong, Y., 2014. 3D finite element modeling for instabilities in thin films on soft substrates. International Journal of Solids and Structures 51, 3619-3632. doi:10.1016/j.ijsolstr.2014.06.023.

Xu, F., Zhao, S., Lu, C., Potier-Ferry, M., 2020. Pattern selection in core-shell spheres. Journal of the Mechanics and Physics of Solids 137, 103892. URL: http://www.sciencedirect.com/science/article/pii/S0022509619310567, doi:https://doi.org/10.1016/j. jmps.2020.103892.

Zhao, R., Kim, Y., Chester, S.A., Sharma, P., Zhao, X., 2019a. Mechanics of hard-magnetic soft materials. Journal of the Mechanics and Physics of Solids 124, 244-263. doi:10.1016/j.jmps.2018.10.008.

Zhao, S., Xu, F., Fu, C., Huo, Y., 2019b. Controllable wrinkling patterns on liquid crystal polymer film/substrate systems by laser illumination. Extreme Mechanics Letters 30, 100502. doi:10.1016/j.eml.2019.100502. 